Modern charge density studies: the entanglement of experiment and theory

Piero Macchi

Department of Chemistry and Biochemistry, University of Bern, Freiestrasse 3, 3012

Bern. Switzerland

piero.macchi@dcb.unibe.ch 


\title{
Modern charge density studies: the entanglement of experiment and theory
}

\begin{abstract}
This tutorial review article is intended to provide a general guidance to a reader interested to learn about the methodologies to obtain accurate electron density mapping in molecules and crystalline solids, from theory or from experiment, and to carry out a sensible interpretation of the results, for chemical, biochemical or materials science applications. The review mainly focuses on X-ray diffraction techniques and refinement of experimental models, in particular multipolar models. Neutron diffraction, which was widely used in the past to fix accurate positions of atoms, is now used for more specific purposes. The review illustrates three principal analyses of the experimental or theoretical electron density, based on quantum chemical, semi-empirical or empirical interpretation schemes, such as the Quantum Theory of Atoms in Molecules, the semi-classical evaluation of interaction energies and the Hirshfeld analysis. In particular, it is shown that a simple topological analysis based on a partition of the electron density cannot alone reveal the whole nature of chemical bonding. More information based on the pair density is necessary. A connection between quantum mechanics and observable quantities is given in order to provide the physical grounds to explain the observations and to justify the interpretations.
\end{abstract}

Keywords: electron density; X-ray diffraction; multipolar model; ab initio calculations; polarized neutron diffraction

\section{Contents}

1. Introduction

1.1 Definitions and physical background

2. Electron density from experiment

2.1 X-ray diffraction

2.2 Other scattering techniques

3. Electron density from theory

3.1 Gas phase calculations 
3.2 Condensed matter

4. Analysis of the electron density

4.1 The quantum theory of atoms in molecules

4.2 Hirshfeld analysis and conceptual DFT

4.3 Fine and coarse grain approaches for the intermolecular interactions

5. Conclusions and outlook

\section{Introduction}

X-ray diffraction has always been deeply interconnected with quantum mechanics. It was in fact soon after Laue's experiment when P. Debye forecasted that this technique could reveal the electronic structure of atoms (1). Although this idea was taken with some scepticism (2) and could not be proven with sufficient accuracy until at least the 1960 's (3) the analysis of X-ray diffraction was anyway challenging quantum physics and chemistry, at least in two areas. One was the physics of light scattering, a new field that originated since the quantum mechanics era. Time has shown that more sophisticated calculations were necessary to explain properly the X-ray atomic scattering form factors of some heavier elements and that required relativistic and multiconfiguration calculations. The other challenge for theory was instead related to chemistry, because X-ray diffraction could reveal in details the geometry of a molecule, therefore giving insights into theories or models for the chemical bonding. As a matter of fact, an accurate molecular geometry is a quantum mechanical observation, no matter if it comes from a theoretical calculation or an experimental measurement.

When later on it became possible to visualize detailed electron density maps (see 
section 1.1), in the form of difference electron densities, theory was again challenged, as not only the precise geometry but also the accurate distribution was now investigated of even minor fractions of electrons around the atoms (localized in the bonding as well as in the non-bonding regions). Indeed, only accurate calculations could provide electron density maps able to explain the experimental findings.

This entanglement of experiment and theory has continued also in the past two decades, when quantum chemical methods, thanks especially to the enormous progresses of the density functional theory (4), has undergone an impressive growth, being now able to describe with sufficient accuracy even very large and complicated chemical systems.

It was in this period when theory and experiment became more closely matched at the level of accuracy of both and therefore the quality of the results obtained improved. In fact, on the experimental side the development of more brilliant X-ray sources (rotating anodes and third generation 'high brightness', 'insertion device design', synchrotrons) and more sensitive and rapid detectors (imaging plates and CCDs) allowed fair comparisons of improved experiments with theoretical methods.

In more recent times, however, the amount of theoretical prior, which is beneficial in modelling of the experimental X-ray diffraction has risen. It is now the experiment which is challenged by theory. Some recent highly accurate experimental results showed that in fact theoretical predictions of even finer details could be confirmed by experimental observation only by using highly accurate data. Moreover, the number of studies that use a combined theoretical/experimental modelling is growing, opening the possibility to optimize so called "X-ray constrained molecular orbital wave functions" or to refine simultaneously the electron, spin and momentum density (see paragraph 2.1.2.1) 
The purpose of this tutorial review is to guide the reader through the methods to obtain electron charge densities, describing the most adopted models, and the interpretation schemes in order to gain some insight into the machinery of modelling techniques and the more sensible applications and interpretations of the experimental results. Already in the above, various technical terms are used and which must be explained to assist the newcomer to the field. That said there are books, admittedly with a research fields focus rather than for students or the newcomer to the field, that cover such material in yet more extensive detail; these are cited below in the appropriate places.

\subsection{Definitions and physical background}

Before starting the discussion of experimental and theoretical methods to obtain accurate electron density mapping, some basic definitions and a short survey of the physics are necessary.

As we know, quantum mechanics has transformed the physical sciences from a deterministic into a probabilistic view. For small particles travelling at high speed, like electrons, it is impossible to simultaneously know their position and momentum (5). We can only know a probabilistic distribution of the electrons in molecules, which means an averaged probability to find any electron at a given position in space. In a system of $N$ electrons and $M$ nuclei the probability of finding any of its electrons at a position $\mathbf{r}_{1}$, regardless of the position of the others, is equal to $\rho\left(\mathbf{r}_{1}\right) \mathrm{d} \mathbf{r}_{1}$ where the corresponding probability is the one electron density ${ }^{1} \rho(\mathbf{r}):(6)$

\footnotetext{
${ }^{1}$ The one electron density is the probability to find any electron at the position $\mathbf{r}$. A two electron density is the probability to find any electron pair at the position $\mathbf{r}$.
} 
$\rho(\mathbf{r})=N \int \Psi_{e l}\left(\mathbf{r}, \mathbf{r}_{2}, \ldots \mathbf{r}_{N} ; \mathbf{R}\right) \cdot \Psi_{e l}^{*}\left(\mathbf{r}, \mathbf{r}_{2}, \ldots \mathbf{r}_{N} ; \mathbf{R}\right) d \mathbf{r}_{2}, \ldots d \mathbf{r}_{N}$

Within the Schrödinger formalism, $\Psi_{e l}$ is the stationary wave function describing the $N$ electrons, at fixed nuclear space coordinates. In equation (1), we are within the BornOppenheimer approximation (7), whereby the electrons and nuclei descriptions are assumed to be independent, and $\mathbf{R}$ denotes the ensemble of nuclear coordinates for the $M$ nuclei in an arbitrary reference frame.

A fundamental piece of information is lost if the one electron density only is known: the correlation among the $\mathrm{N}$ electrons, i.e. how the electrons mutually affect each other. Because of the probabilistic indeterminacy of positions, the electron correlation is also known in a probabilistic form, through more general quantities like the $p$-order density matrices:

$\gamma_{p}\left(\mathbf{r}_{1} \mathbf{r}_{2} \ldots \mathbf{r}_{p} ; \mathbf{r}_{1}^{\prime} \mathbf{r}_{2}^{\prime} \ldots \mathbf{r}_{p}^{\prime}\right)=\left(\frac{N}{p}\right) \int \psi_{e l}\left(\mathbf{r}_{1} \mathbf{r}_{2} \ldots \mathbf{r}_{p} \mathbf{r}_{p+1} \ldots \mathbf{r}_{n}\right) \psi_{e l}^{*}\left(\mathbf{r}_{1}^{\prime} \mathbf{r}_{2}^{\prime} \ldots \mathbf{r}_{p}^{\prime} \mathbf{r}_{p+1}^{\prime} \ldots \mathbf{r}_{n}^{\prime}\right) d \mathbf{r}_{p+1} \ldots d \mathbf{r}_{n}$

where $\{\mathbf{r}\}$ and $\left\{\mathbf{r}^{\prime}\right\}$ are two sets of independent electron coordinates. The first order $(p=$ 1) and second order $(p=2)$ density matrices are certainly the most relevant for the interpretation of the chemical bonding. The one electron density of equation (1) is the trace of the first order density matrix (when $\mathbf{r}=\mathbf{r}^{\prime}$ ):

$$
\gamma_{1}\left(\mathbf{r}_{1} ; \mathbf{r}_{1}^{\prime}\right)=N \int \psi_{e l}\left(\mathbf{r}_{1} \mathbf{r}_{2} . . \mathbf{r}_{n}\right) \psi_{e l}^{*}\left(\mathbf{r}_{1}^{\prime} \mathbf{r}_{2}^{\prime} . . \mathbf{r}_{n}^{\prime}\right) d \mathbf{r}_{2} \ldots d \mathbf{r}_{n}
$$

The off-diagonal terms show, at least in part, the mutual influence of electrons. $\gamma_{1}$ is normalized to the total number of electrons, whereas the second order matrix $\gamma_{2}$

$$
\gamma_{2}\left(\mathbf{r}_{1} \mathbf{r}_{2} ; \mathbf{r}_{1}^{\prime} \mathbf{r}_{2}^{\prime}\right)=\frac{N(N-1)}{2} \int \psi_{e l}\left(\mathbf{r}_{1} \mathbf{r}_{2} \mathbf{r}_{3} . . \mathbf{r}_{n}\right) \psi_{e l}^{*}\left(\mathbf{r}_{1}^{\prime} \mathbf{r}_{2}^{\prime} \mathbf{r}_{3}^{\prime} . . \mathbf{r}_{n}^{\prime}\right) d \mathbf{r}_{3} \ldots d \mathbf{r}_{n}
$$


is normalized to the total number of electron pairs and again the trace gives the two electron density $\rho\left(\mathbf{r}_{1}, \mathbf{r}_{2}\right)$, i.e. the averaged distribution of electron pairs in space. It is clear that most of the chemistry we know depends on these two quantities, the first and second order density matrices, which are quantum mechanical observables.

In equations (1-4) we have not considered an additional variable, which is the spin of the electrons. It is important to be reminded that electrons are Fermions, therefore they obey Fermi-Dirac statistics, which means that two identical spin particles cannot occupy the same position in space (i.e. according to the fundamental Pauli Exclusion Principle (8)), but two opposite spin electrons tend to pair to lower their energy. It is important to recall as well that some multi-electronic systems may be characterized by a given number of unpaired electrons (arising from an odd number of electrons or from a more stable unpaired spin state), therefore producing an excess of electrons in a given spin. While this cannot be identified from eq (1), a more general formulation of the electron density would include the spin as coordinate by extending $\mathbf{r}$ in equations (1-4) to include $\mathbf{x}$, a variable containing the three space coordinates and the spin coordinate. In this way the so-called spin density, i.e. the excess of electron density of one spin, is formally represented by:

$$
\begin{aligned}
& \rho_{\alpha}(\mathbf{r})-\rho_{\beta}(\mathbf{r})= \\
& N\left(\int \Psi_{e l}\left(\mathbf{x}_{1}, \mathbf{x}_{2}, \ldots \mathbf{x}_{N} ; \mathbf{R}\right) \cdot \Psi_{e l}^{*}\left(\mathbf{x}_{1}, \mathbf{x}_{2}, \ldots \mathbf{x}_{N} ; \mathbf{R}\right) d \mathbf{s}_{\alpha} d \mathbf{s}_{2}, \ldots d \mathbf{s}_{N} d \mathbf{r}_{2}, \ldots d \mathbf{r}_{N}-\right. \\
& \left.\int \Psi_{e l}\left(\mathbf{x}_{1}, \mathbf{x}_{2}, \ldots \mathbf{x}_{N} ; \mathbf{R}\right) \cdot \Psi_{e l}^{*}\left(\mathbf{x}_{1}, \mathbf{x}_{2}, \ldots \mathbf{x}_{N} ; \mathbf{R}\right) d \mathbf{s}_{\beta} d \mathbf{s}_{2}, \ldots d \mathbf{s}_{N} d \mathbf{r}_{2}, \ldots d \mathbf{r}_{N}\right)
\end{aligned}
$$

where s are the electron spin coordinates.

It is important to consider that the scattering of photons by electrons is closely connected with the density matrix (equation [2]). In fact, the coherent and elastic (Bragg) X-ray scattering depends entirely on the electron density, the trace of the 
electron density matrix, whereas the incoherent and inelastic (Compton) scattering is produced by the off-diagonal terms. Therefore, the electron density matrix is in principle fully observable, in its diagonal and off-diagonal terms, if Bragg and Compton diffraction are both measured. Moreover, one should consider that a radiation beam of spin active particles, like polarized neutrons, can reveal the excess of electron spin, and hence the spin density.

In this review, we will mainly focus on the one electron density, with only minor reference to the complete density matrix(es), the two electron density and the spin density. The reader is instead referred to some recent literature (9) to gain full insight into the techniques to obtain those functions and their potential applications in chemistry, physics and material science.

At this point, the connection with crystallography is quite clear: the most powerful way to observe scattering is in fact the diffraction from the crystals, better from single crystals rather than by powder diffraction. As anticipated above, the Bragg X-ray scattering intensity distribution is described by the Fourier Transform (hereinafter FT) of the thermal averaged one electron density distribution in a unit cell,

$$
\mathrm{F}(\mathbf{H})=\int_{V}<\rho(\mathbf{r})>e^{2 \pi i \mathbf{H} \cdot \mathbf{r}} d \mathbf{r}=F T<\rho(\mathbf{r})>
$$

where $\mathrm{F}(\mathbf{H})$ is the structure factor, $\mathbf{H}=h \mathbf{a}^{*}+k \mathbf{b}^{*}+l \mathbf{c}^{*}$ is the scattering vector in the reciprocal space, $\mathrm{V}$ is the unit cell volume and $\langle\rho(\mathbf{r})>$ is the thermally averaged electron density distribution (i.e. the convolution between the electron density and the nuclear motion). In principle, equation (6) could be Fourier inverted producing the electron density, as reconstructed from its sampling at discrete points of the reciprocal space (i.e. where Bragg scattering occurs for $n \lambda=2 d \sin \theta)$ : 
$\langle\rho(\mathbf{r})\rangle=\frac{1}{V} \sum_{h, k, l} \mathbf{F}_{\mathbf{h k l}} e^{-2 \pi i(h x+k y+l z)}$

In practice, there are three main complications in making this 'simple' Fourier back transform: 1) the effective sampling of the FT $\rho(\mathbf{r})$ is quite limited, because we cannot measure an infinite number of reflections, but only a finite subset (due to geometrical limitations of the measuring apparatus, to the X-ray wavelength and to the actual intensities of the higher resolution reflections, which are weak); 2) under the kinematic approximation, whereby the scattered X-ray Bragg reflection wave does not interfere with the incident beam wave or other Bragg reflected beam waves, the phases of each $\mathbf{F}_{\text {hkl }}$ vector are not measurable but they are available only from an atomic model; this is particularly cogent for non-centrosymmetric structures where there is no special constraint on the phases (phases of centrosymmetric crystals being necessarily 0 or $\left.180^{\circ}\right)$; 3) the moduli of the structure factors are derived from the experimentally measured intensities, that are of course affected by measurement errors and which naturally propagate through all subsequent calculations.

For these three reasons, we cannot expect to observe an accurate $<\rho(\mathbf{r})>$ from a direct Fourier transform of the structure factor amplitudes, derived from the measured Bragg reflection X-ray intensities. Moreover, the thermally averaged electron distribution is not the static electron density itself which cannot become available unless introducing a model describing the molecular motion. Therefore, as we will see in section 2 , the best way to obtain electron density from experiment is refining a model using the scattered Bragg X-ray reflection intensities for optimizing the parameters.

In general a $F T$, with suitable phases, of the measured structure factor amplitudes would provide an electron density integral or derivative (thermally averaged) property $\mathrm{P}$, either 
scalar or vectorial:

$P(\mathbf{r})=\frac{c}{V} \sum_{h, k, l} H^{n} \mathbf{F}_{\mathbf{h k l}} e^{-2 \pi i(h x+k y+l z)}$

where $c$ is a coefficient, $H$ is the modulus of the scattering vector $(=2 \sin \theta / \lambda) \cdot n=-2$ for the electrostatic potential; $n=-1$ for the electric field; $n=0$ for the electron density and the electric field gradient; $n=+1$ for the gradient of the electron density and $n=+2$ for the Hessian of the electron density and the Laplacian. It should be clear that the limitations due to finite diffraction resolution referred to above affect much more the reconstruction of properties with $n \geq 0$, like the Laplacian of the electron density, and much less those with $n<0$, like the electrostatic potential. However, ripples due to Fourier series termination effects would still affect the map of electric potential constructed with a FT. On the other hand, electron density deformation functions, i.e. calculated from the difference between two sets of structure factors (e.g. observed calculated), would not suffer from the Fourier series termination problems because of mutual cancellation of the positive and negative ripples. This is why the deformation densities have been extremely popular, because they are obtainable using experimentally measured structure factor moduli, using a given model to compute the reference structure factors and the phases to obtain proper structure factor vectors. The most straightforward reference model is the so-called independent atom model (IAM, hereinafter), obtained as the simple superposition of atomic ground state spherical (or spherically averaged ${ }^{2}$ ) densities $\rho_{j}$, so that:

${ }^{2}$ It is very important to recall that the electron densities of many atoms are not spherical in their ground state, for example most transition metals, but also main group p-block atoms. 
$\rho_{\text {unitcell }}(\mathbf{r})=\sum_{j} \rho_{j}\left(\mathbf{r}-\mathbf{r}_{j}\right)$

Where $\mathbf{r}_{j}$ is the position of atom $j$. Noteworthy, IAM is the standard model used to refine crystal structures from X-ray diffraction in conventional crystal structural analyses.

There are several deformation densities in use and here we summarize the most important ones:

(1) Residual Density: it is calculated through a Fourier summation

$\Delta \rho(\mathbf{r})=\frac{1}{V} \sum_{h, k, l}\left(F^{0}-F^{1}\right) e^{-2 \pi i \varphi^{1}} e^{-2 \pi i(h x+k y+l z)}$

For each reflection $h k l, F^{l}$ and $\varphi^{l}$ are the structure factor modulus (amplitude) and phase calculated with a given model, whereas $F^{0}$ is the modulus of the observed structure factor. This density should return the unexplained features of the observation, which could be due to the limitations of the model or otherwise to systematic errors in the experiment.

(2) $X$-X deformation density: using the same kind of Fourier summation as in equation (10), where $F^{l}$ and $\varphi^{l}$ are calculated from IAM high order refinement (i.e. using only data above a given resolution, typically better than $0.7 \AA$ ). Again, $F^{0}$ are the observed X-ray diffraction structure factors. This deformation density should return the main features of the valence electron density, because a high order model is obviously neglecting the effects of the valence electrons (that do not scatter at high diffraction angle).

(3) $X$-N deformation density: $F^{l}$ and $\varphi^{l}$ are calculated with IAM using positional and thermal parameters obtained from a neutron crystal structure refinement. $F^{0}$ are the 
observed X-ray structure factors. As before, this density should return the main distortions of the valence electron density from the spherically averaged distribution, because the neutron model returns very accurate nuclear positions and thermal parameters of the nuclei.

(4) Model dynamic deformation density: here two models are compared through a Fourier summation:

$$
\Delta \rho(\mathbf{r})=\frac{1}{V} \sum_{h, k, l}\left(F^{0} e^{-2 \pi i \varphi^{0}}-F^{1} e^{-2 \pi i \varphi^{1}}\right) k^{-2 \pi i(h x+k y+l z)}
$$

Both models 0 and 1 provide phases and moduli of the structure factors. The aim of this density is to compare two models (defined below) within the limitation of the observed diffraction resolution (as only the measured reflections used to refine the two models are used) and through the smearing of the thermal motion (as both structure factors are computed using the refined atomic displacement parameters).

(5) Static deformation density: this deformation density is not calculated in reciprocal space but in direct space.

$$
\Delta \rho(\mathbf{r})=\rho^{0}(\mathbf{r})-\rho^{1}(\mathbf{r})(12)
$$

The two densities come from two different models, for example two different levels of multipolar expansion (see section 2.1). If model 1 is the IAM and model 0 is a multipolar expansion, then this density should be quite similar to that of the experimental X-X deformation density. Many other static deformation densities could be computed, for example the theoretical multipolar densities (where theoretical can mean calculated for the molecule or for the crystal). 
In figure 1, the main deformation densities for a hydrogen oxalate anion in LHistidinium Hydrogenoxalate (10) are shown.

One of the main purposes of the residual density map is revealing the deficiencies of a given model or the impact of systematic errors of the experimental diffraction measurements. Thus, the residual density of an IAM model (very similar to the X-X or $\mathrm{X}-\mathrm{N}$ deformation densities) should reveal the regions of space where electrons involved in a chemical bond are concentrated, given that this information is obviously absent in the 'simple' structural model. On the other hand, the residual map should appear featureless if the model is accurate enough (see section 2.1). The inspection of the residual electron densities might be particularly difficult in large molecules due either to computational limitations, although with current computing power this is less of a problem, or due to a too-large atomic thermal motion. Moreover, the noise accompanying a residual map could hide some more serious problems; while the residual density may not produce large peaks at some positions, it could anyway reveal some systematic effect. In these circumstances, a statistical analysis of the entire residual density in the unit cell is necessary. Meindl and Henn (11) proposed a statistical analysis to ascertain if only Gaussian noise affects the residual density, see Figure 2. Significant deviations from a Gaussian-type distribution would reveal serious systematic effects, likely due to uncorrected errors in the diffraction data. The remark by Jorgensen et al. (12) on the proper scaling and the effect of corrections to raw diffraction data should be taken into account. 


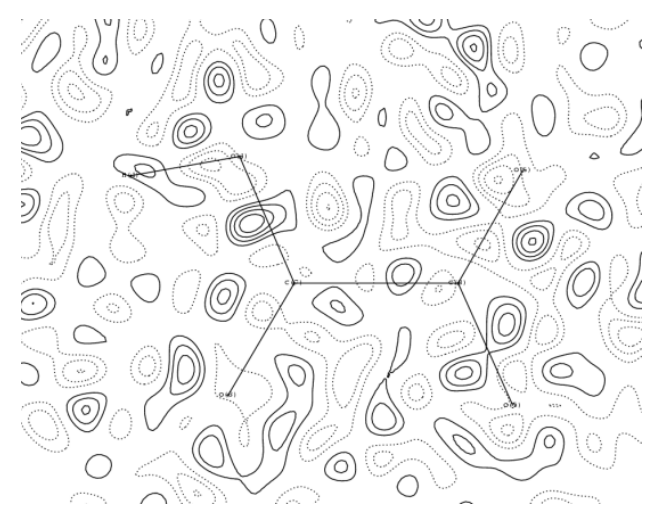

a

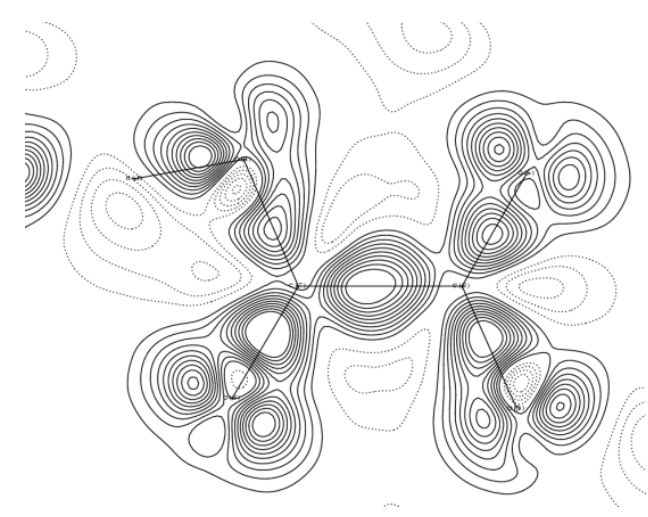

c

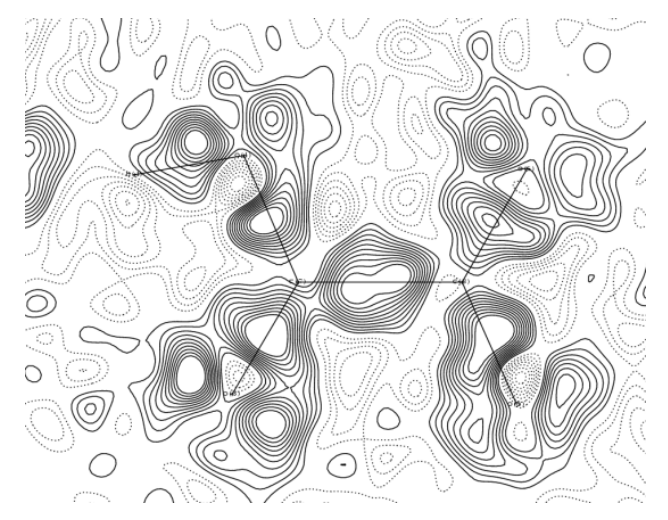

b

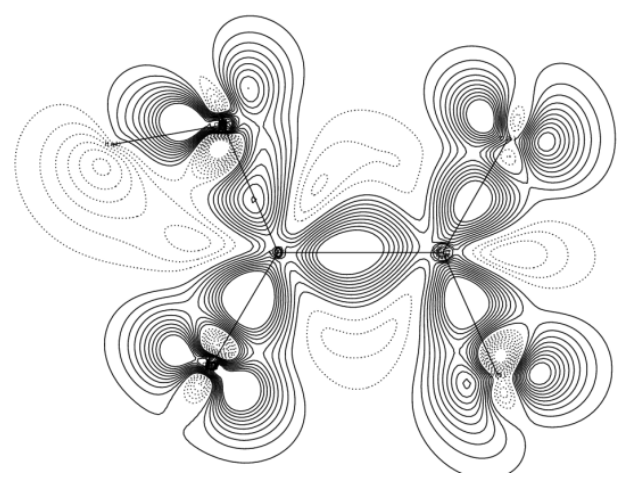

d

Figure 1. The residual density map after multipole refinement (a), the X-X deformation density map (b), the model dynamic deformation density map (c) and the static deformation density map (d) of the hydrogen oxalate ion in L-Histidinium hydrogen oxalate (10), from experimental electron density modelling using data at $100 \mathrm{~K}$, and at $0.45 \AA$ diffraction resolution. All data are included in these Fourier summations. Contours are drawn at $\pm 0.05 \mathrm{e}^{-3}$, with positive electron density contours shown as solid lines, negative electron density contours shown as dotted lines. 


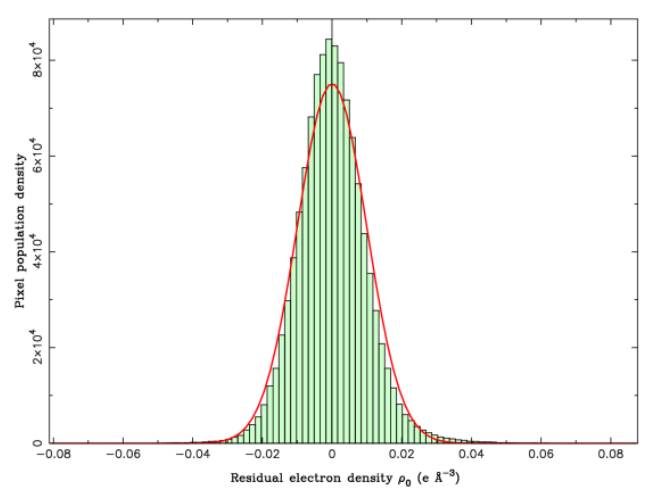

$\mathrm{a}$

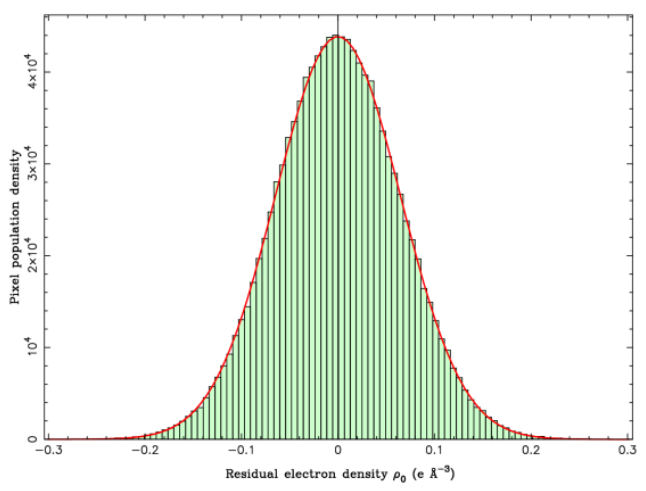

$\mathrm{b}$

Figure 2. Henn-Meindl plots (11) of the distribution of residual electron density pixels for a multipolar fitting against (a) ab initio structure factors (not including thermal motion and experimental errors) and (b) experimental structure factor amplitudes with calculated phases for L-Histidinium hydrogen oxalate (10). Notably, in both cases a Gaussian distribution of the errors is observed, but the FWHM of the theoretical plot is ca. one order of magnitude smaller than the experimental $\left(10^{-2} v s .10^{-1} \mathrm{e}^{-3}\right)$. The small FWHM spread in the theoretical plot is caused by the small differences between the quantum mechanical electron probability density and the multipolar reconstruction. The larger FWHM spread in the experimental plot reveals not only the limitations of the model but also the statistical distribution of experimental errors. 


\section{Electron density from experiment}

When talking about experimental charge density, the more straightforward association is with X-ray diffraction, and often the word "X-ray charge density" is adopted or even "synchrotron X-ray charge density" (13), when a non-conventional i.e. not home source $\mathrm{X}$-radiation is used. We will see in this section the main reasons as to why X-ray scattering is still the most adopted methodology and we will learn how to model electron density from X-ray scattered intensities.

\subsection{X-ray diffraction}

It is well known that the interaction of X-ray photons and electrons produce scattering (elastic or inelastic), whose interpretation could be used to reconstruct the 3D distribution of electrons. However, the correct reconstruction of the direct space electron density image is possible only if the unknown (and in principle nonmeasurable) phases of the scattered photons are somehow obtained, which implies having a model or at least some physical constraints that allow direct calculation of the phases of the structure factors.

\subsubsection{Where is the information?}

As many crystallographers know, the crystal structure of a good quality organic molecular crystal could be refined with an IAM model leaving a residual index of $3 \%,{ }^{3}$

\footnotetext{
${ }^{3} \mathrm{R}_{1}=\Sigma|| F_{\mathrm{o}}|-| F_{\mathrm{c}}|/ \Sigma| F_{\mathrm{o}} \mid\left(\mathrm{F}_{\mathrm{c}}\right.$ are structure factors calculated with the model; $\mathrm{F}_{\mathrm{o}}$ are the observed structure factors)
} 
provided that the sample is cooled to a sufficiently low temperature and that the measurement is carried out with sufficient care. As we discussed above, the structural model that gives such a low residual is actually a very simple one that considers each atom as an independent and spherical scattering center that oscillates harmonically about its nucleus, i.e. according to a restoring force $\mathbf{F}=-\mathrm{k} \mathbf{x}$. Taking into account that the $3 \%$ discrepancy depends also on the statistical errors of the diffraction measurements together with any uncorrected systematic error (such as crystal sample absorption, diffraction extinction effects, unstable with time and uncorrected X-ray source intensity variations, detector uncorrected or residual non-uniformity or instability errors etc.), one can immediately realize that the information on the perturbation of the electron density distribution due to the chemical bonding and the deviation from sphericity effects are very small and therefore it requires highly accurate X-ray diffraction data to be measured. Things might be even worse if heavier elements are present in the molecule under study, because the spherical scattering of their cores would largely dominate the diffraction pattern and a residual index of $2 \%$ or less could be easily obtained upon a simple IAM refinement, making it yet more difficult to obtain the extra information that we are seeking. Coppens et al. (14) proposed as a "suitability indicator" (SI), an inverse measure of the core scattering intensity per unit cell volume ${ }^{4}$. A low $S I(<1)$ is normally calculated for transition metal complexes and could be even lower for intermetallic systems or alloys, especially for structures containing metals of the lower periods of the periodic table.

${ }^{4} S I=\frac{V}{\sum_{j} n_{j, \text { core }}^{2}}$, where $\mathrm{V}$ is the unit cell volume, $n_{j, \text { core }}$ are the number of core electrons of the $j^{t h}$ atom and the summation runs over all atoms in the unit cell 
In Figure 3, we can appreciate the relative importance of core and valence electrons in the X-ray scattering of an organic crystal as a function of the diffraction resolution (measured with the length of the scattering vector, in $\AA^{-1}$ ).

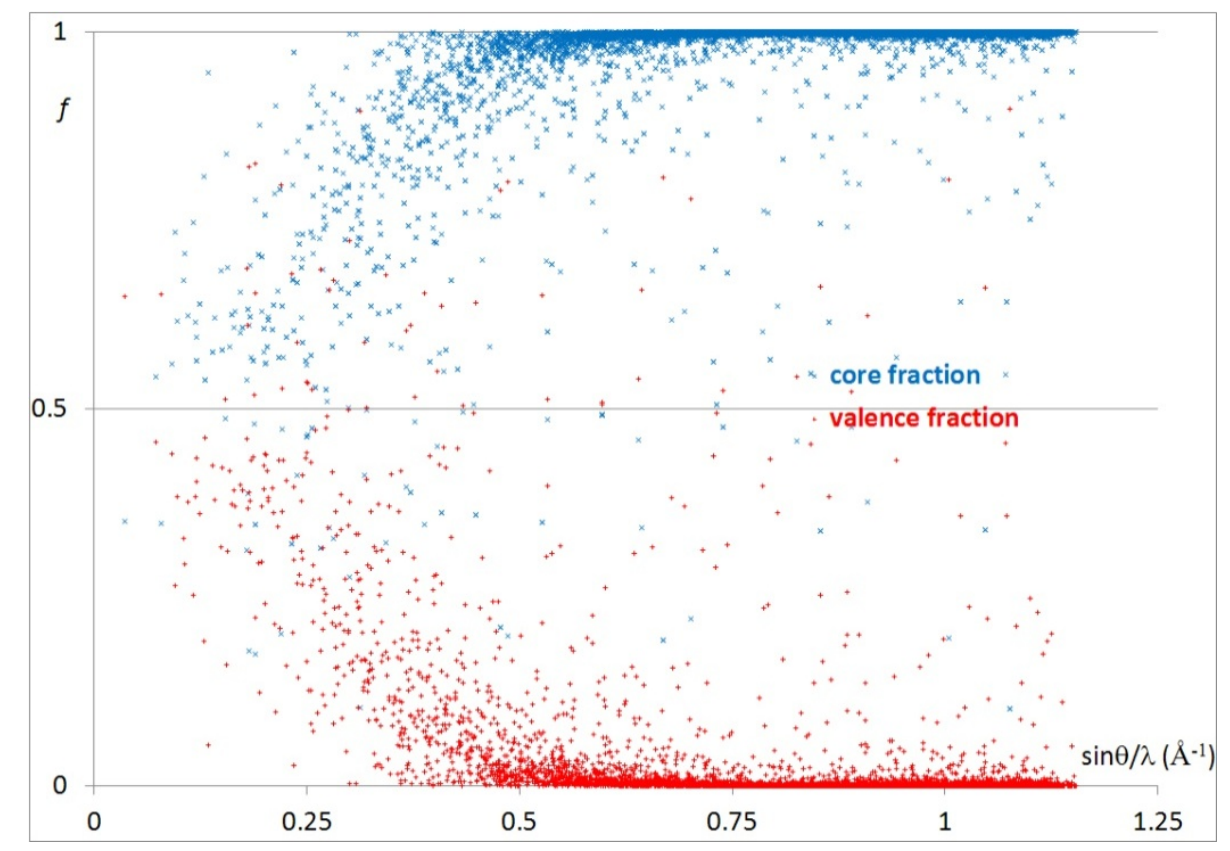

Figure 3. A typical distribution of the core (blue) and valence (red) relative contribution $f$ to the total structure factor, as a function of $\sin \theta / \lambda$. Note that the valence contribution is rarely in excess, apart for a few low order reflections. At high angle most reflections are almost $100 \%$ due to the core density. This simulation is based on a theoretical electron density model for L-Histidinium Hydrogen oxalate (10).

An interesting simulation illustrates the very narrow zone separating a rough and an accurate electron density determination. Let's take an isolated molecular electron density, calculated $a b$ initio with quantum chemical methods at density functional 
theory (DFT) level, ${ }^{5}$ and make a FT in reciprocal space, thus obtaining the theoretical structure factors (defined above), which are of course error free and do not account for the atomic displacements. ${ }^{6}$ If we now fit these data with a simple IAM, the residual factor will be sufficiently good, especially for the high resolution reflections (that are not much affected by the valence electrons), and quite a lot better than what is normally obtained with actual experimental diffraction data.

Table 1. $\mathrm{R}_{1}$ agreement indexes (based on all data) for refinements of some molecules in the gas phase or embedded in a molecular crystals, against theoretical (DFT) or experimental structure factors.

\begin{tabular}{|c|c|c|c|c|}
\hline Compound & Structure factors & Resolution ( $\AA$ ) & IAM & Multipolar model \\
\hline $\begin{array}{c}\text { Hydrogen oxalate } \\
\left(\mathrm{C}_{2} \mathrm{HO}_{4}\right)^{-}\end{array}$ & $\begin{array}{c}\text { Molecular orbital } \\
\text { calculations } \\
(\text { DFT) }\end{array}$ & 0.46 & 0.0155 & 0.0038 \\
\hline $\begin{array}{c}\text { L-Histidinium } \\
\left(\mathrm{C}_{6} \mathrm{H}_{10} \mathrm{~N}_{3} \mathrm{O}_{2}\right)^{+}\end{array}$ & $\begin{array}{c}\text { Molecular orbital } \\
\text { calculations (DFT) } \\
\text { (a) }\end{array}$ & 0.46 & 0.0184 & 0.0033 \\
\hline $\begin{array}{c}\text { L-Histidinium } \\
\text { Hydrogen oxalate }\end{array}$ & $\begin{array}{c}\text { crystal orbital } \\
\text { calculations (DFT) } \\
\text { (b) }\end{array}$ & 0.43 & 0.0171 & 0.0032 \\
\hline $\begin{array}{c}\text { L-Histidinium } \\
\text { Hydrogen oxalate }\end{array}$ & $\begin{array}{c}\text { Experiment on } \\
\text { crystal (10) }\end{array}$ & 0.43 & 0.0494 & 0.0310 \\
\hline $\begin{array}{c}\text { Chromium } \\
\text { hexacarbonyl } \\
\text { Cr(CO) })_{6}\end{array}$ & $\begin{array}{c}\text { Molecular orbital } \\
\text { calculations (DFT) } \\
\text { (c) }\end{array}$ & 0.42 & 0.0122 & 0.0026 \\
\hline $\begin{array}{c}\text { Chromium } \\
\text { hexacarbonyl } \\
\text { Cr(CO) }\end{array}$ & $\begin{array}{c}\text { crystal orbital } \\
\text { calculations (DFT) } \\
\text { (b) }\end{array}$ & 0.46 & 0.0141 & 0.0032 \\
\hline Chromium & Experiment on & 0.43 & 0.0194 & 0.0092 \\
\hline
\end{tabular}

${ }^{5}$ Hereinafter, we refer as theoretical or ab initio the electron density and related properties (like structure factors) which are computed from first principle quantum mechanical methods. The adjective calculated, in association with structure factors, is instead referred to $F_{c}$ computed with a given refined model (for example IAM or multipolar).

${ }^{6}$ We not only consider a temperature of $0 \mathrm{~K}$, but also neglect the zero point vibration that exists at an 'absolute zero' temperature. 


\begin{tabular}{|c|c|l|l|l|}
\hline $\begin{array}{c}\text { hexacarbonyl } \\
\mathrm{Cr}(\mathrm{CO})_{6}\end{array}$ & crystal (15) & & & \\
\hline
\end{tabular}

(a) carried out at B3LYP/6-31G(2d,2p) level of theory.

(b) carried out at periodic B3LYP/6-31G(2d,2p) level of theory.

(c) carried out at BLYP-ZORA/QZ4P level of theory

In Table 1, the results of various structural and electron density refinements of isolated molecules or the same molecule in a crystal, from experiments or from ab initio calculations, are shown. One can observe that for transition metal complexes or in general heavier element molecules the agreement is particularly good already at IAM level. This simply demonstrates that a pure superposition of unperturbed atomic densities (normally referred to as the promolecule), albeit lacking of any physical meaning, is sufficiently close to the exact electron density distribution.

\subsubsection{Modeling}

There are basically two ways to determine an accurate electron density distribution from diffraction data: a) constructing a parameterized analytical function whose Fourier transformation fits the observed Bragg reflection intensities or moduli; b) calculating the electron density at each point in space from a FT of the structure factors, based on the observed scattered intensities and using the phases from an IAM refinement. Of course, the numerical solution b) could eventually be fitted with a function in order to obtain an analytical description. There is in principle no special restriction on the analytical functions to be adopted, however practical considerations orient the choice towards quantum mechanical functions, known to reproduce quite well the behavior of the electron distribution in particular regions. For example, a Slater Type Orbital (hereinafter STO) is a function like: 
$[2 n !]^{-1 / 2}(2 \zeta)^{(n+1) / 2} r^{n-1} \exp (-\zeta r)$

which is able to reproduce exactly the electron-nucleus cusp condition (16), that other functions, like Gaussian or Lorentz, can only approximate, by using a suitable combination of a number of these functions. Using STO would be a way to allow the experimentally reconstructed function to obey a given physical constraint, thus introducing a bias. It is important to note that STOs are nodeless, unlike the hydrogenlike Schrödinger equation derived electron orbitals. Energy optimized STOs are easily calculated for all atoms in their ground state using Roothaan's equation (17) and orthonormal atomic orbitals $\phi_{l}$ as basis sets:

$\phi_{l}=\sum_{j=1}^{m} c_{j}\left[2 n_{j}\right]^{-1 / 2}\left(2 \zeta_{j}\right)^{\left(n_{j}+1\right) / 2} r^{n_{j}-1} \exp \left(-\zeta_{j} r\right)$

$\phi l$ are therefore expanded in a series of STO functions of the same symmetry, but not necessarily of the same principal quantum number $n$. In fact, the 1s orbital is normally expanded in terms of several functions, of type $1 \mathrm{~s}, 2 \mathrm{~s}$, $3 \mathrm{~s}$ etc. The same holds true for all other orbitals, which eventually result in all being orthogonal to each other. This means that their densities can be simply summed to produce the total electron density. The most famous set of Roothan-Hartree-Fock wave functions is that tabulated by Clementi and Roetti (18). These functions are the ideal starting points to fit the experimental diffraction data, although these are not mandatory. One could in fact use just one function for each atomic orbital, the so called best single- $\xi$ orbitals (19).

There are other physical constraints that could be taken into account when refining a 
model, for example the positivity of the electron density ${ }^{7}$ at all points in the unit cell, the Hellmann-Feynman theorem (electric forces at nuclei vanish), (20) the electroneutrality of a crystal, etc. In principle one could also apply constraints based on observed properties of the crystal, for example the piezo-electric constants, however this has never been taken into account. ${ }^{8}$

In general, we can classify the electron density determinations in two categories: a) reconstruction within quantum mechanical constraints; b) non-quantum mechanical reconstructions. The first category is by far the most adopted and successful, although the amount of quantum mechanical prejudice or prior that we must introduce could even become "absolute". In this case, the density is fully biased toward theoretical quantum mechanics, thus without using any information from experimentally observed $\mathrm{X}$-ray structure factor intensities and therefore cannot be called experimental.

\subsubsection{Quantum mechanical modeling}

As anticipated, a quantum mechanical modeling is one where, at least to some extent, information from quantum chemistry is used. This is true even for conventional crystal structural analysis, based on IAM refinements: in fact, the atomic scattering factors, used for the calculated structure factors, come from quantum mechanical wave functions computed for isolated atoms in their ground state using integro-differential

\footnotetext{
${ }^{7}$ It is well known that although representing the distribution of negative charges, the electron density is a probability distribution, therefore must be positive by definition.

${ }^{8}$ To the best of the author's knowledge.
} 
Hartree-Fock equations or even Dirac-Fock equations. ${ }^{9}$ These include the relativistic effects, which are particularly important for the heaviest elements, because of the "contraction" occurring to the orbitals of the core electrons.

The de-convolution of the temperature induced atomic displacements from the thermally averaged electron density is also based on the quantum mechanical harmonic oscillator and the lattice dynamics as introduced by Born and Huang (21) and developed by Willis and Pryor (22). The reader is referred to a recent review for more details (23). Here we just recall that the atomic form factors can be approximated with the product of the electronic and the nuclear density probability function, so that equation (6) can be approximated with:

$\mathrm{F}(\mathbf{H})=\int_{V}<\rho(\mathbf{r})>e^{2 \pi i \mathbf{H} \bullet \mathbf{r}} d \mathbf{r} \approx \sum_{i} n_{i} f_{i}(\mathbf{H}) T_{i}(\mathbf{H}) e^{2 \pi i \mathbf{H} \bullet \mathbf{r}}$

$T_{i}(\mathbf{H})$ is the Debye-Waller factor that is the $F T$ of the atomic probability density function undergoing thermal motion, and $p_{i}(\mathbf{U})$ that depends on the atomic displacement vector $\mathbf{U}$. Within the harmonic oscillator approximation, $p_{i}(\mathbf{U})$ is a three dimensional Gaussian function (isotropic or anisotropic) and $\mathbf{U}$ is a second order tensor (or a simple scalar in case of a symmetric, isotropic probability function). This is the typical modelling used by crystallographers for crystal structure refinement of small molecules or inorganic compounds. For an accurate treatment, a more extensive model could be refined, which includes the anharmonic motion. The most adopted is the socalled Gram-Charlier (24) expansion of the harmonic Debye Waller factor. In this case,

\footnotetext{
${ }^{9}$ The solution of these equations is not an eigenvalue problem as the Roothaan equations described above, therefore the solution, which is less approximate, is numerical. Nevertheless, atomic form factors can be retrieved from these numerical solutions.
} 
a generalized $\mathbf{U}$ could be defined (25) including a third and fourth order tensor. Quite important is that the Gram-Charlier expansion requires a larger number of parameters (10 for the third order expansion and 15 for the fourth order). This implies large correlation among the parameters of a least square refinement and therefore a higher diffraction resolution is necessary, depending also on the mass of the atoms involved (26). It should be taken into account that Gram Charlier expansion could produce nonpositive probabilities, which are of course lacking physical sense. Therefore, the results of Gram Charlier refinements should be carefully scrutinized.

When the purpose is the determination of an accurate electron density distribution, then the amount of quantum mechanical prior becomes larger. Accurate here means that the function is closer to the exact quantum mechanical one electron density defined in equation (1) than that computed with a simple IAM. For this goal, one should define atomic or molecular orbitals and tentatively refine their occupancies. The first attempts to refine this type of model were proposed in the late 1960's and early 1970's. This task however was rather complex. In fact, the many diffraction intensities that one could obtain from a single crystal were not so easily transformed into the desired information, given that the Fourier inversion approach would fail to reconstruct an accurate electron density (of course they could reconstruct a rough electron density function, sufficient to achieve a crystal structure solution). Thus, the first successful works used the same idea beyond crystal structure refinement that is the partitioning into a given number of atom projected functions, whose parameters are refined against the observations. The socalled "Stewart atom" (27) (i.e. the atom centered projected molecular densities that best fit the total density), is still adopted in quantum chemistry (28) as one of the most interesting partitioning of molecules, valid not only for modeling diffraction experiments. Of course this projection requires a set of atomic bases, i.e. a series of 
atom-centered functions that must be appropriately chosen to guarantee completeness.

Although the IAM is also based on atom-centered functions (see equation (9)), they are not obtained from the projection of the total density. On the other hand, although lacking of any physical meaning, the IAM molecular density distribution has in fact found some important applications in theoretical chemistry and in the analysis of the electron density as we will see in Section 4.2.

Thanks to the work of R. F. Stewart (29), K. Kurki-Suonio (30), P. Coppens (31), N. K. Hansen (32) and F. L. Hirshfeld (33) a theoretical framework was developed to describe the deformation density (i.e. the atom centered electron density which deviates from sphericity). The method they developed is called the multipolar model that retains the same atomic approximation of IAM, but with a larger flexibility. In fact, it was proved that it is possible to expand the total molecular electron density in terms of atom centered functions. For the sake of completeness and closure, their angular parts were chosen to be spherical harmonics (i.e. the same functions used for atomic orbitals). The atom centered multipolar expansion is a better approximation of the Stewart atom (27) than the IAM. With atom-centered functions one loses the possibility to distinguish between single-center and multi-center electron density, because all contributions are projected onto single centers. However, the atom centered expansion is simple enough because it retains a connection with intuitive chemistry, providing atomic functions that describe the total number of electrons of an atom and their aspherical distribution, from which a set of atomic (29c) and molecular (34) multipolar moments is easily derived.

The authors mentioned above proposed different interpretations of the multipolar expansion. That mostly adopted is the Hansen and Coppens model (32). Here the electron density of an atom $i$ is expanded in three main components: the core density 
(typically a spherical term with frozen population), a spherical valence density (with variable population and contraction/expansion $\kappa$ ) and a deformation valence density (with variable multipole coefficients and expansion/contraction $\kappa^{\prime}$ ):

$$
\rho_{i}(\mathbf{r})=P_{i, \text { core }} \rho_{i, \text { core }}(\mathbf{r})+P_{i, \text { valence }} \kappa_{i}^{3} \rho_{i, \text { valence }}(\mathbf{r})+\sum_{l=0, l_{\max }}\left[\kappa_{i}^{\prime 3} R_{l}\left(\kappa_{l}^{\prime} \mathbf{r}\right) \sum_{m=0, l} P_{l m \pm} y_{l m \pm}(\mathbf{r} / r)\right]
$$

The parameters $P_{l m \pm}, P_{\text {valence }}$ and $\kappa$ are typically refined within a least squares procedure versus the X-ray diffraction data, together with positional and thermal parameters, as in a normal crystal structure refinement. The spherical valence density is separated from the deformation density which also contains a spherical monopole (when $l=0$ ). This allows one in principle to refine at least two monopoles for the valence density orbital distribution (a feature useful especially for split valence atoms like transition metals).

The various multipolar models found their applications through proper software developed over the years, for example XD2006 (35), VALRAY (36), Molly (37), MoPro (38), JANA2006 (39). The Stewart formalism, although proposed earlier (29), has been less adopted also because of the more restricted distribution of the software associated with this method, VALRAY. Stewart's formalism can be expressed as: ${ }^{10}$

$$
\rho_{i}(\mathbf{r})=P_{i, \text { core }} \rho_{i, \text { core }}(\mathbf{r})+\sum_{l=0, l_{\max }}\left[R_{l}\left(\zeta_{l} \mathbf{r}\right) \sum_{m=0, l} P_{l m \pm} y_{l m \pm}(\mathbf{r} / r)\right]
$$

Where, normally, atomic $P_{\text {core }}$ parameters are refined for each electronic shell of an atom but only one monopole for the valence shell(s) is defined.

One of the most important advantages of the formalism proposed by Coppens was the

\footnotetext{
${ }^{10}$ Noteworthy, refining an exponent $\xi$ or the expansion/contraction coefficient $\kappa$ is basically identical.
} 
usage of local coordinate systems: each set of atomic multipoles is defined with respect to a dedicated reference system which is totally independent from the unit cell setting and from the reference systems of all other atoms. In fact, in most other crystallographic applications there is no need to describe an atom with a reference system different from that of the unit cell. For example, special positions in a given space group cause some constraints on the coordinates or thermal parameters of atoms, easily handled using the reference system of the unit cell. On the other hand, the intuition by Hansen and Coppens was that there are in general two different, but equally important references in molecular crystals: one is the orientation of the molecule with respect to the unit cell; the other is the internal coordinates of the molecule (i.e. a definition of each atom within the molecule). The two references are rarely coincident, as molecules normally crystallize with much lower symmetry than their point group symmetry, therefore there is no coincidence between the inertial /symmetry axes of the molecule and the crystallographic directions. More importantly, this choice has indirectly anticipated the work which is characterizing most of the modern applications of charge density analysis. That is the exportability of a set of multipolar parameters (40) from one atom of a given functional group (that can be refined or calculated with high accuracy because it belongs to a simple system chosen as reference) to an equivalent atom, however belonging to a more complex system that cannot be so accurately refined or calculated. This idea requires that each atom is defined independently with respect to a local coordinate system, defined using other atoms of the molecule. This approach not only guarantees the exportability of the multipole parameters but it allows one also to define restraints or constraints on chemically equivalent atoms that are not crystallographically equivalent, for example if a pseudo symmetry is present in the molecule, but it does not coincide with an actual crystallographic symmetry. This 
allows one to refine a smaller number of parameters and to impose local symmetries in

keeping with idealised molecular symmetries.

Table 2. The typical parameters and functions of a multipolar model.

\begin{tabular}{|c|c|}
\hline Parameter / Function & Remarks \\
\hline $\begin{array}{l}\text { Scale factor } \\
\qquad \mathrm{K}\end{array}$ & $\begin{array}{l}\text { It is normally refined, but it would highly correlate with the total number } \\
\text { of electrons, unless this is constrained (via the electro-neutrality } \\
\text { constraint). As an alternative, the scale factor could be calculated based } \\
\text { on a high diffraction angle data with spherical atom refinement, and then } \\
\text { kept frozen when the valence monopoles are refined without constraints. } \\
\text { The first option is more common and creates less problems. }\end{array}$ \\
\hline $\begin{array}{l}\text { Atomic fractional } \\
\text { coordinates } \\
\mathrm{x}, \mathrm{y}, \mathrm{z}\end{array}$ & $\begin{array}{l}\text { They are normally refined together with the multipolar parameters. This } \\
\text { does not work for } \mathrm{H} \text { atoms, as they would highly correlate with the } \\
\text { dipolar functions. Thus } \mathrm{H} \text { atoms must be positioned based on some } \\
\text { external method, like theoretical calculations, neutron diffraction, } \\
\text { average positions based on similar species, vibrational spectroscopy etc. }\end{array}$ \\
\hline $\begin{array}{l}\text { Atomic displacement } \\
\text { parameters } \\
\mathrm{U}_{\mathrm{iso}}, \mathrm{U}_{\mathrm{ij}}, \mathrm{U}_{\mathrm{ijk}}, \mathrm{U}_{\mathrm{ijkl}}\end{array}$ & $\begin{array}{l}\text { The } \mathrm{U}_{\mathrm{ij}} \text { tensor is normally refined apart for } \mathrm{H} \text { atoms (same } \\
\text { considerations as for } \mathrm{x}, \mathrm{y}, \mathrm{z} \text { above). A treatment of the thermal motion } \\
\text { beyond the harmonic approximation is possible }\left(\mathrm{U}_{\mathrm{ijk}}, \mathrm{U}_{\mathrm{ijk} \mathrm{l}}\right) \text {, but it } \\
\text { requires yet higher resolution diffraction measurements to avoid a strong } \\
\text { correlation within parameters (41). The positivity of the probability } \\
\text { distribution of the ADPs calculated from this model should be checked. }\end{array}$ \\
\hline $\begin{array}{l}\text { Atomic Core population } \\
\qquad P_{\text {core }}\end{array}$ & $\begin{array}{l}\text { Difficult to refine, but technically possible when quality diffraction data } \\
\text { are available. For more sophisticated treatments of the core electrons, } \\
\text { including full multipole expansion, see the text. }\end{array}$ \\
\hline $\begin{array}{l}\text { Spherical valence } \\
\text { population } \\
P_{\text {valence }}\end{array}$ & $\begin{array}{l}\text { Refinement of this parameter gives an enormous improvement to a } \\
\text { model. }\end{array}$ \\
\hline $\begin{array}{l}\text { Aspherical (deformation) } \\
\text { valence population } \\
\qquad P_{l m}\end{array}$ & $\begin{array}{l}\text { These parameters define the shape of the deformation density around } \\
\text { each atom. A standard model implies refinement up to hexadecapole } \\
\text { level for heavier elements, in particular metals of the d-block; octupole } \\
\text { for main group atom; dipole for } \mathrm{H} \text { atoms. More flexible models are } \\
\text { welcome, if the number and accuracy of the diffraction data allow. }\end{array}$ \\
\hline $\begin{array}{l}\text { Radial scaling of the } \\
\text { spherical valence density } \\
\kappa\end{array}$ & $\begin{array}{l}\text { This is a fundamental parameter that gives an estimate of the contraction } \\
\text { / expansion of the valence shell. It is in principle an atomic parameter, } \\
\text { but more often it is collectively refined for chemically equivalent atoms. }\end{array}$ \\
\hline $\begin{array}{l}\text { Radial scaling of the } \\
\text { deformation valence } \\
\text { density } \\
\kappa^{\prime}\end{array}$ & $\begin{array}{l}\text { In principle a different scaling for each multipole radial density is } \\
\text { possible, but very difficult to obtain convergence. More frequently, a } \\
\text { single } \kappa \text { ' for all multipoles is refined (again grouping chemically } \\
\text { equivalent atoms). }\end{array}$ \\
\hline $\begin{array}{l}\text { Diffraction extinction } \\
\text { coefficients } \\
\varepsilon_{i j}\end{array}$ & $\begin{array}{l}\text { Unlike X-ray absorption of the sample, X-ray diffraction extinction } \\
\text { cannot be corrected during diffraction data processing treatment, given } \\
\text { that a model for the structure factors is necessary. Therefore an } \\
\text { extinction refinement should be carried out against the diffraction data, } \\
\text { at least isotropically, to check if extinction effects are indeed visible }\end{array}$ \\
\hline
\end{tabular}




\begin{tabular}{|c|l|}
\hline & $(42)$. \\
\hline$R_{l}(\mathbf{r})$ & $\begin{array}{l}\text { Radial density functions for the deformation electron density. In } \\
\text { conventional multipolar refinements these are single STO functions, } \\
\text { representing the best single- } \xi \text { functions for an orbital (43) }\end{array}$ \\
\hline$\rho_{i, \text { core }}(\mathbf{r})$ and $\rho_{i, \text { valence }}(\mathbf{r})$ & $\begin{array}{l}\text { Spherical or spherically averaged densities from Roothan Hartree-Fock } \\
(18) \text { or Dirac Fock (44) wave functions (each orbital is expanded in a } \\
\text { number of STO functions). }\end{array}$ \\
\hline
\end{tabular}

It is important to stress that a multipolar expansion of the electron density is possible to construct models not only based on experimentally measured X-ray intensities. For example, Stone (45) proposed a multipolar expansion of the theoretical electron density, calculated from a molecular wave function. The same approach was used also to expand the dipolar polarizability (46). Both expansions are intended to reduce the electrostatic and induction energies of two interacting molecules into simple atom-atom pair wise terms. We will see in section 4.3 that these ideas found applications also when analyzing electron densities from experiment.

One important limitation of a multipolar model is the level of the expansion. It is clear that the larger is the expansion the smaller will be the error. However, expansion in terms of higher multipoles could have practical problems because it requires a large number of parameters and creates convergence problems for their least squares refinement. It is normally assumed that a reflections : parameters ratio of 10 is necessary, otherwise the correlation would be so large that the uncertainty on each parameter would be too large, even enormous. Given that in general, for charge density studies, diffraction data are collected up to a diffraction resolution of $0.5 \AA$, the number of parameters that could be refined is limited to a maximum of 60 per atom. In fact, assuming an atomic volume of ca. $18 \AA^{3}$, the reciprocal volume would be ca. $0.055 \AA^{-3}$, contained ca. 600 times in a sphere of radius $1 / \mathrm{d}=2 \sin \theta / \lambda=2.0 \AA^{-1}$. Taking into account 3 fractional coordinates and 6 components of the $U_{i j}$ tensor per each atom, there could be sufficient parameters for a multipolar expansion at least up to $l=6$ (49 
additional parameters). However, there are some severe problems in this simplified, 'back of the envelope', calculation: a) not all reflections are actually observable, especially at high diffraction angle; b) in the evaluation of $18 \AA^{3}$ per atom, the hydrogen atoms are not included (having a volume of only 1-2 $\AA^{3}$ ); c) an important warning was given by Destro and Roversi (47): the diffracted intensities are not homogeneously distributed among all reflections and some parameters do not contribute at all to some reflections, therefore the calculation should be more conservative. Having these three warnings in mind, a more sensible estimation of what measurements are required is that an X-ray diffraction data collection up to a resolution of $0.5 \AA$ (assuming that at least $80 \%$ of the reflections are above a minimal threshold of intensity limit, usually taken as $2 \sigma(\mathrm{I}))$ allows refinement of all atomic parameters up to hexadecapole level $(l=4)$ for all heavier elements, but hydrogen atoms are limited to just monopole and dipole because the deconvolution of their charge density and atomic displacement parameters is particularly difficult, which is a major reason why neutron diffraction could be employed. This X-ray diffraction data collection could be considered as a conventional or minimal requirement experiment to obtain a charge density distribution. Of course, if the crystal type and quality, the radiation or the detector (or all of them) allow a data collection at a yet higher resolution (better than $0.5 \AA$ ) one could safely expect to obtain an even more accurate charge density description model and perhaps to extract more information from it, e.g. higher accuracy for weaker bonding electron densities.

To illustrate how the multipolar model works, we consider a step by step refinement for some simple molecule, whose theoretical electron density has been calculated with a quantum chemical approach (at B3LYP/6-31G(2d,2p) level (48)) and then Fourier transformed into structure factors (and by including the molecule in an arbitrary unit cell 10x10x10 $\AA^{3}$, for a small enough molecule, and assuming no symmetry i.e. P1 
symmetry), without taking into account thermal motion and random experimental errors of the X-ray diffraction intensities. In Figure 4, we see the residual electron density after carrying out refinements against theoretical electron density derived structure factors calculated for an isolated benzene molecule, as the example, and then increasing the level of the multipole expansion from the IAM up to a multipolar expansion truncated at hexadecapole level. The residual density around the $\mathrm{H}$ atoms is cleared up after the refinement of the dipolar density. In fact there is only one, large, polarization for the $\mathrm{H}$ atoms, which is that in the direction of the $\mathrm{C}-\mathrm{H}$ bond, easily described by just one dipole function on the $\mathrm{H}$ atom (directed as $\mathrm{C}-\mathrm{H}$ ) and then finally adjusted with the final $\kappa$ refinement. On the other hand, the $\mathrm{sp}^{2}$ hybridized $\mathrm{C}$ atoms require additional functions, in particular one octupole function that is quite typical for such a trigonal stereochemistry.

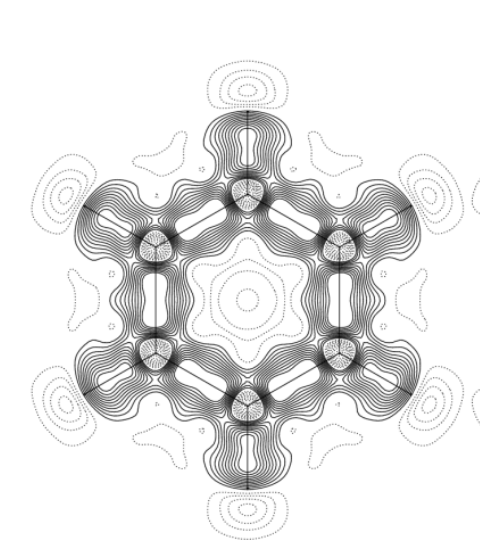

a

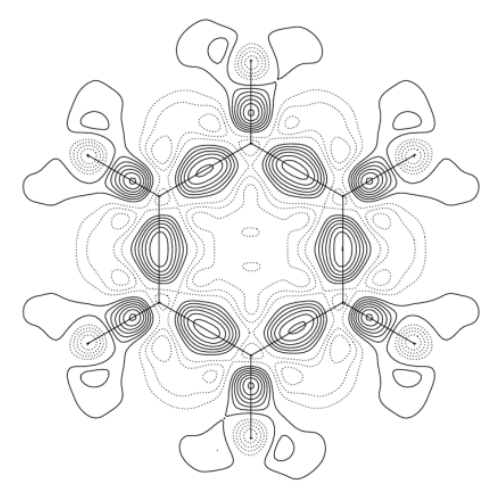

d

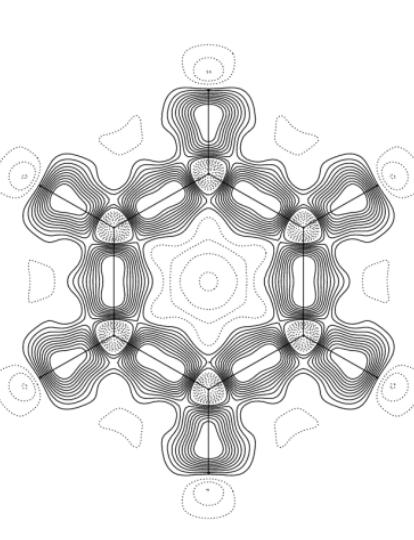

b

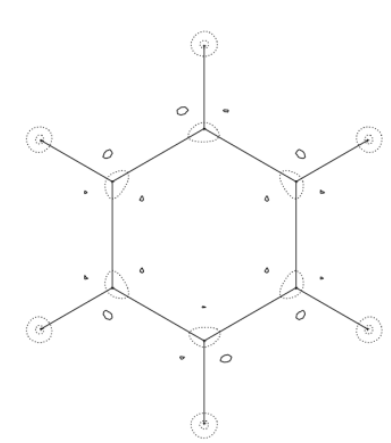

$\mathbf{e}$

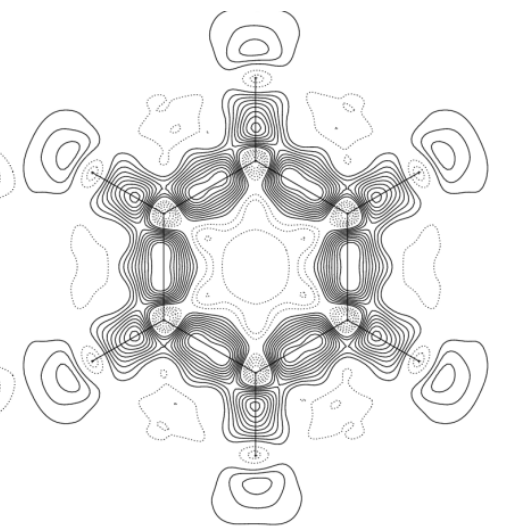

C

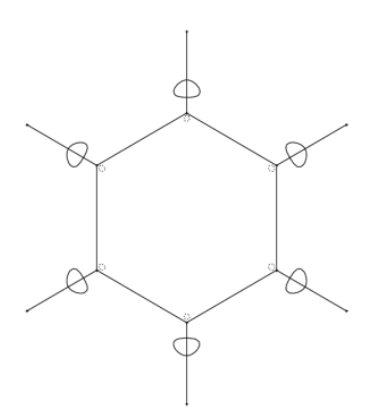

f 
Figure 4 Residual electron densities in a benzene molecule, from multipolar fitting of the theoretical electron density derived structure factors at the B3LYP/6-31G(2d,2p) level. a) Residual density after IAM refinement $\left(\mathrm{R}_{1}=0.0234\right)$; $\left.\mathrm{b}\right)$ residual density after monopole refinement $\left(l=0 ; \mathrm{R}_{1}=0.0221\right)$; c) residual density after dipole refinement $(l$ $\left.\left.=1 ; \mathrm{R}_{1}=0.0195\right) ; \mathrm{d}\right)$ residual density after quadrupole refinement $\left(l=2 ; \mathrm{R}_{1}=0.0135\right)$; e) residual density after octupole refinement $\left(l=3 ; \mathrm{R}_{1}=0.0051\right)$; $\mathrm{f}$ residual density after full multipole refinement $\left(l=4\right.$ and refinements of $\kappa$ and $\left.\kappa^{\prime} ; \mathrm{R}_{1}=0.0031\right)$. The $\mathrm{H}$ atoms are expanded only up to $l=2$. Electron density contour levels are as depicted and defined in Figure 1. A hexadecapole refinement $(l=4)$ would give results very similar to $l=3$ with only minor improvements $\left(\mathrm{R}_{1}=0.0048\right)$. Notice the gradual reduction i.e. improvement of the $\mathrm{R}_{1}$ for each extra level of charge density description as well as the improvement i.e. disappearance in the residual electron density features. Thus, by monitoring both, it can be directly seen that the X-ray diffraction intensities do indeed have the information to describe the electron density in this fine level of detail. 
We will now consider a more complicated structure, such as the octahedral metal complex $\mathrm{Cr}(\mathrm{CO})_{6}$. Here, the main difference is the presence of a fourth period atom, having some d-electrons. Describing a transition metal is also complicated by the double nature of the valence: the energy levels of the $n$ s and $(n-1) \mathrm{d}$ electron orbitals are very close, and that means that both are available with almost equal probability for electron occupation and thereby for the proper combination of atomic orbitals to produce molecular orbitals. Indeed, the electronic configuration of a transition metal in its ground state is also peculiar: most atoms have $n \mathrm{~s}^{2}(n-1) \mathrm{d}^{\mathrm{x}-2}$ where $\mathrm{x}$ is the total number of valence electrons; a few atoms have $n \mathrm{~s}^{1}(n-1) \mathrm{d}^{\mathrm{x}-1}$ or $(n-1) \mathrm{d}^{\mathrm{x}}$ electronic configurations. This demonstrates the rather similar energy of the corresponding states. But these could however be highly perturbed in the electric field of the ligands (neutral or anionic), especially if the metal is positively charged (an oxidation state +2 very commonly produce a $\mathrm{d}^{\mathrm{x}-2}$ configuration).

From Figure 5, we learn that for a transition metal the multipolar model must necessarily extend up to the hexadecapole level $(l=4)$. In fact, the electron probability density of a d-orbital can be exactly expanded in multipolar terms as a summation of a monopole, a quadrupole and a hexadecapole term. This arises from a mathematical closure property of spherical harmonics, whose product is a linear combination of other spherical harmonics and in particular the summation runs over harmonics of order $\mathrm{L}=l$ $+l^{\prime}, \mathrm{L}=l+l^{\prime}-2 \ldots$ etc. (where $l$ and $l^{\prime}$ are the orders of the two harmonics that are multiplied). Thus the electron probability density of a p orbital (which is associated with an $l=1$ spherical harmonic) is described by a quadrupole and a monopole term (coming from the product of two $l=1$ harmonics). As each orbital type would produce a different combination of spherical harmonics, in principle from the refined multipolar coefficients, one could retrieve an atomic orbital population by inverting the matrix 
which transforms electron orbitals into multipoles. However this is in general impossible because the total electron density comes from molecular orbitals where the individual orbital components are no longer orthogonal to each other because of the mixing with orbitals of other atoms in the complex. With transition metals, however, under the hypotheses of a low overlap with the ligand orbitals - the same principle justifying the basis for the Ligand Field Theory (49) - this mathematical transformation would work. In fact, Coppens et al. (50) introduced the method whereby electron orbital coefficients from multipolar refinements of a transition metal atom could be obtained. There is an additional observation which is possible after the examples shown in Figures 4 and 5. In fact, because of the molecular symmetry and the atomic site symmetry, there could be special constraints on the multipoles. The $6 / \mathrm{mmm}$ molecular symmetry of benzene implies that all $\mathrm{C}$ atoms are identical and therefore share the same set of multipoles, if the local coordinate system is identically oriented. The same holds true for the six hydrogen atoms of benzene, of course. On the other hand, the symmetry elements on which some atoms lie impose the constraint that some multipoles cannot be present, because they would violate the site symmetry. For example, if an atom lies on a mirror plane of the molecule, it cannot bear a dipole perpendicular to that plane, because it would be positive one side and negative on the "mirror symmetric" side, which is obviously impossible. This reasoning is particularly cogent for the $\mathrm{Cr}$ atom in $\mathrm{Cr}(\mathrm{CO})_{6}$, lying on an octahedral high symmetry site (see Figure 5e) which allows only an hexadecapolar function together with the spherically symmetric monopole. A collection of the symmetry constraints pertaining to atoms on a special site is summarized in ref. (30e). 

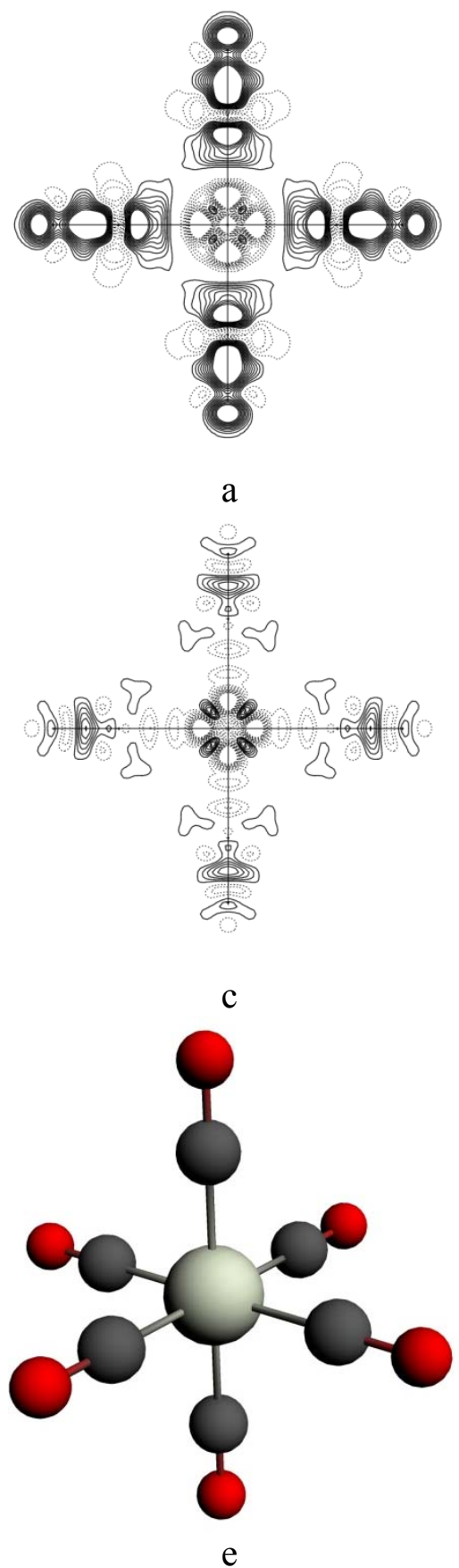
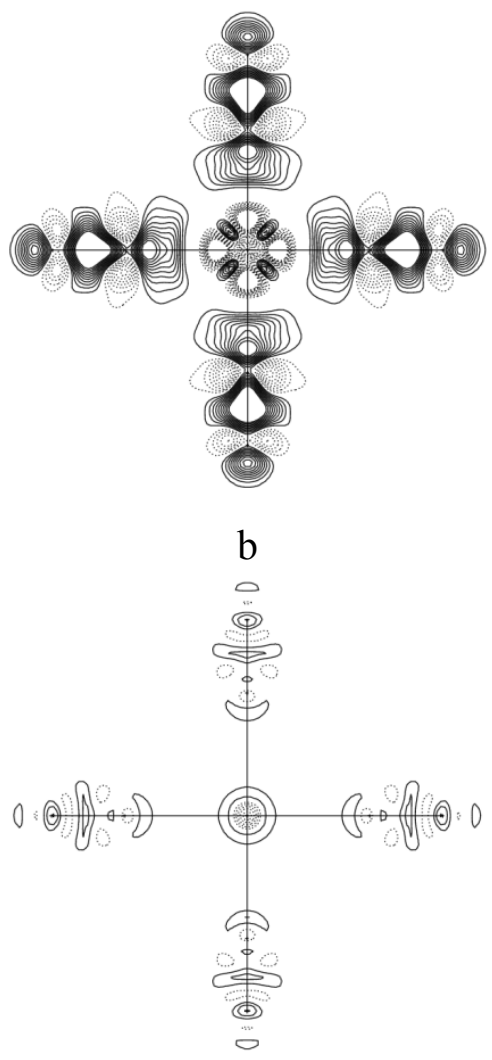

d

Figure 5 Residual electron densities in $\mathrm{Cr}(\mathrm{CO})_{6}$, from multipolar fitting of the theoretical electron density derived structure factors (calculated at a B3LYPZORA/QZ4p level of theory). a) Residual density after IAM refinement $\left(\mathrm{R}_{1}=0.0122\right)$; b) residual density after monopole refinement $\left(l=0 ; \mathrm{R}_{1}=0.0109\right)$; c) residual density after quadrupole refinement $\left(l=2 ; \mathrm{R}_{1}=0.0065\right)$; $)$ residual density after full multipole refinement $\left(l=4 ; \mathrm{R}_{1}=0.0026\right)$. e) molecular structure of $\mathrm{Cr}(\mathrm{CO}) 6$. At all multipole levels, $\kappa$ and $\kappa^{\prime}$ are refined. Electron density contour levels as in Figure 1. 
As anticipated above, the radial description of the atomic density is rather important. The choice of atomic wave functions borrowed from quantum chemistry gives a very good initial estimate, but not necessarily a perfect solution to describe the accurate electron density distribution. In fact, atomic electron densities undergo contractions or expansions from their ground state form, due to interactions with other atoms in a molecule or in a crystal. The electron density deformation is qualitatively predictable: for example, an atom in an electric field will polarize the electron density depending on the direction and magnitude of the applied electric field vector and on the polarizability of the atom. The main problem is that the electric field experienced by an atom is not homogeneous; therefore, each point in space is polarized in a different way. Of course, an atom involved in many different chemical bonds will be anisotropically distorted. Moreover, each part of the atomic electron density has a different response to the applied electric field: core electrons are obviously less polarizable because they are more bound to the nucleus. Last but not least, the polarization depends on the oxidation state and the charge of the atom. In general one could expect that anions are larger than cations, as an excess of electron density makes the electron density cloud larger.

Within the multipolar model, the way to account for a homogeneous expansion or contraction of the electronic cloud of an atom is by modifying the exponent of the STO function used to describe the core or the valence of a given atom, which is easily applied through the $\kappa$ parameters. Much more difficult is taking properly into account the anisotropic deformation due to the chemical bonding. In fact, even within the same electronic shell, one must consider the possibility of different expansion/contractions in different directions. This could be estimated in several ways, but the price would be to significantly increase the number of parameters of the model. A larger flexibility is obtained assigning to each function in the multipolar expansion of equation [15] an 
independent radial function (51):

$$
\rho_{i}(\mathbf{r})=P_{i, \text { core }} \rho_{i, \text { core }}(\mathbf{r})+P_{i, \text { alence }} \kappa_{i}^{3} \rho_{i, \text { valence }}(\mathbf{r})+\sum_{l=0, l_{\max }}\left[\sum_{m=0, l} \kappa_{i m}^{\prime 3} R_{l m}\left(\kappa_{l m}^{\prime} \mathbf{r}\right) P_{l m \pm} y_{l m \pm}(\mathbf{r} / r)\right]
$$

It is obvious that this implies many more parameters, because each multipole $l m l$ has a different radial function (thus $l$ - and $m$-dependent) and $\kappa^{\prime}$ scaling, at variance from the multipole model of equation 15 where radial functions are only $l$-dependent and often they are constrained to be the same for all multipoles, thus being both $l$ and $m$ independent. One should take into account that this ultra-flexible model is intended to pick up the $0.3 \%$ (i.e. 0.003 ) portion in the residual of an agreement factor $\mathrm{R}_{1}$, which remains unexplained after a "conventional" multipolar refinement (Table 1 and Figures 4-5). In most practical examples, however, this approach cannot be used because it requires too many data and or too much accuracy, versus what is usually available. The $0.3 \%$ residual spread is easily lost in the imprecisions of data corrections. The refinement of the model from equation [17] against experimental data could be more stable if the $m$-dependent radial functions are rigidly maintained, that means without any $\kappa^{\prime}$ refinement and the radial functions are taken from calculations of a number of prototype atoms and then stored in a database.

A lower level of flexibility would be that of using a different expansion/contraction for each multipolar level, thus using the parameters $\kappa^{\prime}$ as they actually appear in equation [15], instead of imposing $\kappa^{\prime}=\kappa^{\prime} l$ for each $l$.

Research in this direction is still on-going and one could expect some improvements in the next few years, whereby attempts are being made to reduce any large correlations among variables in this kind of model, and which hampers a full applicability at present. 
While equation [17] is intended to improve the flexibility of the radial functions for valence electrons, in some recent works the role of the core electrons has also been deeply investigated. The possibility to visualize distortions of the atomic cores has been proposed in the past, but with few applications. More recently, instead, Scherer, Iversen and co-workers (52) have shown that even minor features of the polarisations of core electrons are visible if accurate diffraction data are measured, even from powder diffraction, giving as a side product almost perfect thermal parameters, otherwise affected by some unexplained electron density feature. Refining core electron density within a multipolar model is not particularly different from refining the valence electron densities: the principal problems are the very small extents of core deformations and the very fine diffraction resolution that would be necessary to refine them properly.

The core charge density refinement could be carried out in the following ways:

(1) minimal: refining the atomic core monopole populations in a typical multipolar refinement, that means making $\mathrm{P}_{\text {core }}$ in equation [15] a variable (notably this is often the standard in VALRAY (36) refinements);

(2) semi-flexible: refining a scale $\kappa_{\text {core }}$ factor together with the monopole population, allowing therefore a contraction/expansion of the core itself;

(3) flexible: refining a full set of multipoles (even up to hexadecapole) for the core electrons, starting from the orbitals of the atomic wave functions.

(4) extremely flexible: refining different sets of multipoles and contraction factors for each electronic shell of the core for atoms of the third period or higher (thus one set of multipoles for K shell, one for L-shell etc.).

With all these possible models, equation [15] becomes: 


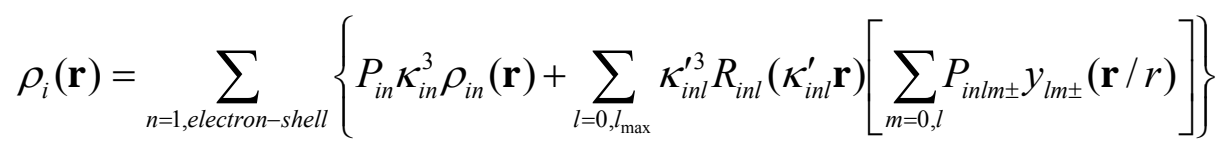

with a different degree of flexibility depending on which recipe (1)-(4) is used. It is also obvious that equation [18] could be merged with [17] making all the radial functions $l$ and $m$-dependent. Practical considerations make the extremely flexible model (4) very rarely usable, because the number of parameters would be enormous. An example is the seminal work of Fischer et al. (52) on $\alpha$-Si, where the very high symmetry and the simplicity of the compound reduce the number of variables even for the most flexible models. In more complex systems, one could use a simplified scheme (3): apart from (pseudo)symmetry considerations, one could also take into account the kind of deformations that are theoretically possible for a core. For atoms of the second period, the cores do not contain $d$ orbitals and the through bond ${ }^{11} 1 \mathrm{~s}-3 \mathrm{~d}$ mixing should be very small, therefore one would not expect very large multipolar contributions above dipole (1s-2p mixing). On the other hand, $2 \mathrm{~s}$ and $2 \mathrm{p}$ orbitals of $3^{\text {rd }}$ period atoms could mix with $3 \mathrm{~d}$, at least in part, producing some more significant contributions of higher multipoles. As a matter of fact, in $\alpha$-Si the refinable octupole and hexadecapole of the K shell of $\mathrm{Si}$ returns very small values (modelled against theoretical wave function parameters). Nevertheless, the very flexible refinement showed a significant contraction of the corresponding valence multipoles and significant figures also for the octupole of the L shell. ${ }^{12}$

${ }^{11} 1 \mathrm{~s}$ and $3 \mathrm{~d}$ are obviously orthogonal in the isolated atoms.

${ }^{12}$ Also the L-hexadecapole is not negligible, although it cannot be produced by s-d or p-d mixing. We can only explain this as a pure $d$ contribution that should give an idea of the total amount of d-polarization in Si. 
As discussed above, the atom centered multipolar expansion is the simplest possible in terms of interpretation and so far scientists have forgiven the limitation of this model, in return for the reduced computational costs (which are of the same order of magnitude of a conventional IAM refinement). This advantage has allowed this field to make enormous progress and to demonstrate the feasibility of charge density experiments and their potential usage.

On the other hand, a larger precision of the modelling (for example the radial function refinement method of Koritsanszky and Volkov (51)) would require much larger computation not only for the refinements but also for the calculation of electron density derived properties (see section 4). For this reason, other methods could become at this point competitive, like the refinement of molecular orbital wave functions against diffracted X-ray intensities. Many authors have tried to propose these kinds of calculations, of course facing the problem that the Hamiltonian of a Schrödinger equation cannot directly contain the measured X-ray intensities. A brilliant solution was proposed by Jayatilaka and coworkers (53), who connected the Hamiltonian and the diffracted intensities by means of a Lagrangian multiplier. This means imposing a constraint to the wave function: the wave function is calculated in such a way that on the one hand it minimizes the electronic energy (the normal variational principle) and on the other hand it minimizes the difference between the FT of the calculated electron density and the observed structure factors. The Lagrangian multiplier is set before the calculation and it determines which minimization is more important (energy or structure factor difference). In this way one obtains a so called "X-ray constrained" wave function out of a self consistent calculation, where neither energy nor electron density are self-consistent, unless the Lagrangian multiplier is set to zero (i.e. observed structure factors are not used). Despite the fact that the quantum mechanical meaning is, strictly 
speaking, lost, this approach has received enormous attention especially because it gave access to a number of correlated properties that are available only if a wave function is known, whereas they cannot be calculated exactly from an electron density function.

An alternative procedure is the direct refinement of the density matrix elements, which implies going well beyond simple Bragg scattering and including Compton X-ray profile measurements. This subject, albeit extremely interesting, is beyond the scope of this tutorial review, but the reader is referred to a recent review of the methods of this part of the field (51a) for more information.

\subsubsection{Non quantum mechanical modelling}

As anticipated above, orbital functions are not strictly mandatory to describe the electron density distribution, as in general orbitals are not necessary to solve the Schrödinger equation. An analytical function is also not mandatory, in fact, as we saw above, the $F T$ of the observed structure factors could in principle produce the electron density at each point in space. However, as already discussed, this would not be a suitable method for the determination of the electron density distribution. Therefore, other approaches have been proposed, which are based on different ways to reduce the ripples that would otherwise occur from a simple $F T$.

For example, the Maximum Entropy Method (MEM) (54) is a technique based on information theory, which was originally introduced in the field of radio-astronomy to extract signals from stars out of noisy backgrounds (55). MEM uses the information entropy to find the most likely distribution of a numerical function like the electron density distribution over the ensemble of all points in a unit cell. It is necessary to 
define an information entropy S (56), to be maximized so as to achieve the least biased interpretation consistent with the experimental data:

$$
S=-\sum_{j=1}^{N_{p}} \rho_{j} \ln \left(\frac{\rho_{j}}{\rho_{j}^{\text {prior }}}\right)
$$

where $\mathrm{N}_{\mathrm{p}}$ is the total number of points in the unit cell, $\rho_{j}=\rho\left(\mathbf{r}_{j}\right)$ is the value of the ED in the pixel $\mathrm{j}$ centred at $\mathrm{r}_{\mathrm{j}}$ and $\rho_{j}^{\text {prior }}=\rho^{\text {prior }}\left(\mathbf{r}_{j}\right)$ is the corresponding value for a convenient but ideally arbitrary prior reference electron density. The prior electron density could be a uniform electron density distribution or that calculated from a simple IAM model, or even a multipolar electron density if the aim of the MEM study is trying to improve the multipole model itself. Maximization of the information entropy, however, would simply produce a homogeneous electron density in the whole unit cell, because in this essentially trivial form of the mathematics it is lacking of any physics based constraint. If constrained to fit a set of observed (or simulated) X-ray structure factors, the MEM procedure produces a more suitable electron density distribution. MEM has often been adopted in electron density studies in order to overcome possible ambiguities of the multipolar model, especially due to limitations of the radial functions. The model independent nature of MEM is however questionable because of the role of the prior information in the definition of the information entropy (57).

On the other hand, MEM has some disadvantages because the electron density which is obtained is not analytical, which substantially reduces the calculations of the electron density properties unless trying to fit the numerical values with proper functions. Moreover, the electron density is thermally smeared, as that coming from a direct FT of the measured X-ray intensity data. This is a limitation, in general, because the main purpose of an electron density study is the static function (the same normally estimated 
via a theoretical calculation). Important is also that the structure factor phases, the scale factor K (defined in Table 2) the anomalous dispersion and the extinction effects are not self consistently calculated. All these corrections to the data must be applied in advance, using for example a preliminary IAM model (59a).

The basic aspects of the application of the MEM approach in crystallography are summarized in the book by Coppens (31), while a discussion of most recent methodological improvements $(57,58)$ and applications of the MEM, in particular to molecular crystals of biological interest, may be found in (59). Software for MEM refinement and analysis is available, for example BayMEM (60). Other software is necessary for a full topological analysis (see section 4.1) of numerically reconstructed densities, for example EDMA (61), Integrity (62) or Bader (63).

It is interesting that MEM has been widely used in crystallography as a method to solve crystal structures especially when data are not so easily interpreted, for example from powder diffraction. This has stimulated many electron charge density studies, as of course powder diffraction seriously limits the application of conventional multipolar models, although the combination of a Rietveld refinement using a flexible model is technically possible (39). On the other hand, the (stated) power of MEM is the ability to reconstruct the function even when the information is substantially hidden in the observations. Limitations of MEM are however due to the termination of the data and, in powder diffraction, to the basically impossible challenge in correctly extracting structure factors amplitudes in a severely peaks overlapping situation, where 'equipartitioning' is not adequate especially at the needed highest diffraction resolutions, as well as the modelling of the X-ray background (64). 
MEM has sometimes been criticized because of artefacts produced in the resultant electron density reconstructions, for example the occurrence of electron density maxima at non-nuclear positions (65). However, it should be kept in mind that any method could produce artefacts due to some approximations and assumptions, like the use of orbital functions, the atomistic approximation etc. In this sense, no model can be considered bias-free.

\subsection{Other scattering techniques}

Although X-ray diffraction is by far the most adopted technique, we should keep in mind also that neutron or electron scattering does provide information on the electron density. Neutrons have been widely adopted in the past to obtain precise and independent estimations of nuclear positions and thermal motion, to be used as the IAM reference for the experimental deformation density maps. Later on this method was gradually left behind as the multipolar model proved satisfactory to bypass the ambiguity of refining atomic positions and their individual thermal motions together with deformation electron density parameters. As a matter of fact a perfect multipolar model would produce atomic xyz and $\mathrm{U}_{\mathrm{ij}}$ parameters close enough to those coming from the refinement based on neutron data. Nevertheless, neutron diffraction has remained a core approach for a precise determination of the thermal parameters for $\mathrm{H}$ atoms, simply not possible with X-rays, which is especially important in studies on the medium-strong hydrogen bond. In fact, although X-ray diffraction methods have been proposed to refine the positions of $\mathrm{H}$ atoms, for example the polarized $\mathrm{H}$ atom model of Stewart $(29 a, 66)$, these are normally less accurate than with neutron diffraction data, and as stated above do not provide $\mathrm{U}_{\mathrm{ij}}$ tensors. On the other hand, a combination of polarized $\mathrm{H}$ 
atom and spectroscopic information has been successfully proposed and implemented, especially by Destro and Roversi (67). More recently, a new approach has become available, which solves most of the problems, making the use of neutrons unnecessary, though taking advantage of substantial theoretical information. In fact, the optimization of molecular crystal geometries under periodic boundary conditions has now become quite easy and reliable. Therefore, the precise coordinates of $\mathrm{H}$ atoms, at the same level of accuracy as from neutron diffraction, are available at relatively lower investigator effort (much lower than involved in undertaking a neutron diffraction experiment at a centralised facility). At the same time, the determination of hydrogen $U_{i j}$ values is possible combining the external molecular motion (quite easily determined from an accurate X-ray diffraction) and vibrational frequencies available from spectroscopy, like in the method proposed by Destro (67), or otherwise from the calculations themselves, albeit they necessarily require a correction for the anharmonic motions of the $\mathrm{H}$ atoms (68).

Despite the fact that the usage of neutrons can basically be considered as obsolete, there is still a very good reason to carry out neutron diffraction experiments, namely using polarized neutrons (PNs). In fact, PNs interact with the electronic spins of atoms and their diffraction provides information on the spin density (69). Moreover, a new methodology was proposed, which combines the refinement using X-ray and polarized neutrons diffraction data, using a unified Hansen-Coppens formalism. Research is now concentrated in the direction of combining Bragg X-ray scattering, PN and Compton scattering profiles to produce a joint electron, spin and momentum density refinement (51a). 


\section{Electron density from theory}

A full quantum chemical treatment is beyond the scope of this review. We will limit our descriptions here to briefly describe the most adopted and reliable methods to obtain theoretical electron densities, and focusing especially on those of more crystallographic applications like the calculations under periodic boundary conditions that obviously are the case in a crystal.

\subsection{Gas phase calculations}

In this section, we refer to the determination of electron density in isolated molecules therefore ignoring any external perturbation, such as the electric field of other molecules in a crystal.

Most of the theoretical methods adopted to solve self-consistently the Schrödinger equation (70) allow the definition of an electron density matrix and therefore provide an electron density distribution. The most widely adopted methods are based on Molecular Orbital wave functions, obtained from a linear combination of atomic orbital basis sets. Noteworthy, these basis sets are typically constructed with Gaussian type functions, mimicking a STO function. Stewart showed that the Gaussian expansion introduces a relatively negligible error (27), which is however quite concentrated at the nucleus.

The reader is referred to some specialized books to evaluate the different methods, basis sets and their reliability (71). From the point of view of the electron density it is obvious that a better wave function, obtained from a multi-configuration (and perhaps multistate) calculation, would provide a more accurate electron density distribution.

However, the challenge of estimating a "perfect" wave function is the computational 
overhead. Given the increasing complexity of the molecular systems that are more and more being investigated, the density functional theory (4) (DFT), especially in the formalism of Kohn-Sham orbitals (4b), has grown as the best standard method, given its inherent simplicity and the relatively lower computational costs. The reason is that although based on a single configuration it is supposed to partially include the effects of the electron correlation, albeit in a semi-empirical way. Electron correlation cannot be accounted for at the Hartree-Fock level, where only one electronic configuration is calculated. Electron correlation requires that interaction of more electronic configurations is explicitly considered.

Many approximated functionals have been proposed at similar computational costs, but which would require an accurate testing of their performances. Normally, pure generalized gradient approximation functionals ${ }^{13}$ or hybrid functionals ${ }^{14}$ give the best performances in terms of molecular geometries, vibrational frequencies, binding energies and activation barriers. Similar results are expected if the electron density is analyzed, although systematic comparisons in terms of electron density parameters are limited to a molecular dipole moment treatment (71).

In general, it is obvious that the theoretical methods should include a sufficient amount of electron correlation (72) in order that the electron density approaches as much as possible the experimental results. Therefore, functionals able to mimic at best the effects of the electron correlations are those that provide the more reliable molecular geometry and electron density distribution. In this respect, the hybrid functionals are

\footnotetext{
${ }^{13}$ The exchange functional is expressed in a local form which includes the gradient of the electron density, which is different from the Local Density Approximation in which the Exchange functional only depends on the electron density.

${ }^{14}$ The Exchange functional is combined with some amount of exact Hartree-Fock exchange.
} 
normally the best candidates. More recently, dispersion corrected functionals have been introduced. If the dispersion is included in a non-variational way (applying a correction to the DFT variational energy) then this will certainly improve the molecular geometry but not directly the electron density. On the other hand, in approaches like the Coulomb attenuation method (73) (CAM) that modifies the Hamiltonian itself, the dispersion corrected density is itself variational, i.e. it is calculated by minimizing the energy.

While one can regret that little work has been done in order to compare the various functionals using electron density as benchmarks, one can report interesting observations from the literature that could be further exploited:

(a) Volkov and Coppens (74) reported that multipolar models refined against theoretical structure factors were closer to the experiment when hybrid functionals (B3LYP) were employed, rather than for pure Hartree-Fock. This preliminary observation guarantees that the electron correlation introduced via the functional is visible in the experimental data. Moreover it is notable that in all DFT methods, it is the electron density and not the electronic wave function to be optimized, which therefore anticipates a better performance.

(b) Koritsanszky (75) reported that finer features of DFT functionals could not be revealed if experimental conditions were simulated. This means that the current experimental techniques would not be able to further scrutinize the quality of a theoretical approach.

(c) Santos and Macchi reported (76) that CAM-B3LYP (73) returns atomic density parameters and molecular polarizabilities that are closer to much more expensive coupled-cluster techniques (like CCSDT), where single double and triple excitations are 
accounted for. This means that the most accurate DFT functionals may replicate, at a much lower cost, the performances of the least approximated quantum chemical methods.

(d) The X-ray constrained wave function methods proposed by Jayatilaka (53), and discussed in section 2.2.2.1, can be carried out at various levels of theory including the experimental information (weighted by the Lagrangian multiplier). In the absence of any constraint of the X-ray diffraction intensities, the method is identical to a normal molecular wave function calculated with a given Hamiltonian. If the Hamiltonian is the simple Hartree-Fock operator, than the SCF wave function is a pure Hartree-Fock. If the Lagrangian multiplier is activated, instead, the wave function is no longer purely variational. The method becomes a special kind of DFT. As a matter of fact the difference between an X-ray diffraction constrained Hartree-Fock and DFT wave function (and the corresponding densities) calculation is much smaller than that obtained with the unconstrained methods.

\subsection{Condensed matter}

For the crystallographic community, more interesting than gas phase calculations are theoretical methods for calculations of periodic electron densities, mimicking crystal structures. For practical and historical reasons, DFT methods are by far the most adopted. Hartree-Fock periodic wave functions are rarely employed nowadays and available only in some software codes. Perturbation theories applied on Hartree-Fock wave functions are in principle possible, but at enormous computational costs (77).

The basis set is a very important component in periodic DFT calculations. Most of the software codes make use of plane waves (PWs) instead of orbitals. PWs are naturally periodic functions: 
$f_{G}(\mathbf{r} ; k)=\frac{1}{\sqrt{\Omega}} \exp [i(\mathbf{k}+\mathbf{G r})](20)$

$\mathbf{G}$ is the reciprocal lattice vector, $\mathbf{r}$ is the direct space vector, and $\Omega$ is the unit cell volume, and $\mathbf{k}$ is the reciprocal space vector. PWs are inherently periodic and continuous. PWs are the researcher's elective choice especially for crystalline metals, given the delocalized nature of the electrons in these solids. PWs provide some advantages: they are orthonormal, they are not atomic dependent and convergence of the Kohn-Sham equation could be computationally very fast. It is normal procedure, however, to omit the core electron density, which is more difficult to describe with PWs and easily simulated within the functional itself, but this prevents the analysis of core electron density distortions.

An alternative to PWs are the atom-centered orbital functions, typically Gaussian-type, an analogue of the molecular orbital approach (78):

$$
\chi_{i}\left(\mathbf{r}_{i}\right)=\sum_{j=1, M} c_{i j} N_{l m}\left(\alpha_{i j}\right) y_{l m}\left(\mathbf{r}_{\mathbf{i}}\right) \exp \left(-\alpha_{i j} r_{i}^{2}\right)
$$

where $\mathbf{r}_{\mathrm{i}}$ is the vector distance from the nucleus $i, y_{l m}$ are spherical harmonics, $N_{l m}$ are normalization coefficients, $c_{i j}$ are contraction coefficients and $\alpha_{i j}$ are Gaussian exponents, $M$ is the number of Gaussian primitives of angular momentum components $l$ and $m$. In the crystal, for the orbital wave functions, the Bloch condition must be applied. Problems arise from the inherent incompleteness of these kinds of basis sets and the diffuse atomic orbitals that could lead to quasi-linear dependence of the crystal orbital. On the other hand, for molecular crystals rather than inorganic solids, the major advantage is a very immediate comparison between the isolated gas phase molecule and the molecule in the crystal as nominally the very same basis set could be adopted. 
A software for estimating the crystal orbitals periodic wave function is CRYSTAL09 (78), and which calculates Hartree-Fock or DFT wave functions. CRYSCOR (77) can calculate perturbation corrections to the Hartree-Fock energy.

For PW calculations, solid state physics researchers have a variety of software programs available, among which that are well used are QuantumExpresso (79), Vasp (80) and Wien2K (81). 


\section{Analysis of the electron density}

So far, we have discussed methods to obtain accurate electron density distributions, either from theory or from experiment. We will now focus on the most important applications of the analysis of the obtained electron density distribution. Most of them allow one to extract information on the chemical bonding, intra- or inter-molecular, its nature and strength. Other applications are related to molecular or crystal properties, directly or indirectly dependent on $\rho(\mathbf{r})$.

\subsection{The Quantum theory of atoms in Molecules}

Richard Bader has dedicated his entire scientific career to the development of a theory based on the electron density distribution with the aim to produce a self-consistent understanding of chemistry (82). At variance from other quantum mechanical partitioning of the wave function, Bader provided an alternative way to identify atoms in molecules, based on a rigid space partitioning based on the field gradient of the electron density distribution. Interestingly, this topological partitioning of the electron density distribution coincides with a quantum chemical definition of atoms, based on Schwinger's quantum action principle (83) (a differential statement which connects the time and the transformation of quantum mechanical observables in kinematical and dynamical variations).

Although QTAIM would not be useful for fitting X-ray scattered intensities, it provided the possibility to partition all molecular properties into an individual atomic basis, including the energy itself and to analyze the chemical bonding with new eyes. In fact, Bader was able to show that an accurate analysis of the electron density, and 
derivatives, could shed light on the nature of the chemical bonds, a perspective alternative to the traditional analyses of the molecular wave functions (84).

All these results were not accepted without severe criticism (85), nevertheless, in the past three decades, the Quantum Theory of Atoms in Molecules (hereinafter QTAIM) has achieved a tremendous momentum. One of the major reasons was that it gave, for the first time, a common platform for theoreticians and experimentalists together, otherwise entrenched in their very different points of view. Notable is the fact that in the 1970s and 1980s, comparisons were made between experimental deformation electron density maps (therefore including the thermal motion of atoms) of molecules in crystals and theoretical static deformation densities (without thermal motion of the atoms) of in effect gas phase molecules. On the other hand the development of an analytical description of the experimental electron density on the one side, and the QTAIM on the other, gave a common language to speak about the very same quantities (e.g. the topological analysis of the static electron density) and to compare theory and experiment without prejudice.

In this tutorial review, we cannot present a detailed overview of QTAIM. The reader is referred to more specialized and comprehensive books $(82,86)$ or review articles of the research field on this subject. Here we limit the discussion to some illustrative examples.

\subsubsection{Topological analysis}

The basic idea of Bader and co-workers (87) is the possibility to partition the electron density into atomic domains (or basins) and approach in real space the chemical bonding problem. An atomic domain is defined as a region of space in which all the 
electrons which are bound to the nucleus. Of course, the kind of binding forces chosen for the partitioning define atomic basins having different meanings. Apart from the classical gradient field $\nabla \rho(\mathbf{r})$ ie the gradient of the electron density introduced by Bader (87), similar partitioning styles have been proposed but based on the electric field (88) (the total electrostatic force acting on a charge) or the Eherenfest force (89), (the total force acting on an electron of the system by the nuclei and all the remaining electrons).

We will limit our discussion to the "classical" QTAIM, i.e. based on $\nabla \rho(\mathbf{r})$ partitioning, which generates some very important loci:

(a) The nuclear attractor: a stationary point of the electron density corresponding to a nuclear position obviously ignoring the zero point motion that exists even at absolute zero temperature. ${ }^{15}$ Derivatives at this point are not defined due to the cusp, ${ }^{16}$ however in a Gaussian approximation, the point would be characterized by three independent and negative curvatures of the electron densities. In some controversial cases, local attractors (called non-nuclear maxima, NNM) were found in positions very far from a given nuclear site, normally in regions of low electron density, in particular in some

\footnotetext{
${ }^{15}$ Sometime it is erroneously said that electron density maxima and nuclear positions are not coinciding, for example to explain the systematic shortening of bonds to hydrogen atoms in Xray diffraction refined crystal structure models. This interpretation is not correct: even if extremely polarized the electron density generated by STO functions is necessarily maximal at the nuclear position. The large and apparent shortening of $\mathrm{X}-\mathrm{H}$ bond distances ( $\mathrm{X}=$ any atom) is due to the fact that in order to fit extremely polarized and anisotropic electron densities from unpolarized and spherical scattering factors, the easiest way is shifting the pseudoatom center in the direction of the bond. The same holds true if we use polarized but still spherical scattering factors for $\mathrm{H}$ atoms, which is the default option in most of the commonly adopted computer programs.

${ }^{16}$ If we do not take into account the finite size of the nucleus.
} 
metal solids, like $\mathrm{Be}(90)$, or in small $\mathrm{Li}$ or $\mathrm{Na}$ atom clusters (91). The interpretation of these points is as yet unresolved (92), although in some cases their occurrence has been demonstrated to be a pure artifact of the reconstruction model (65). According to Martín Pendás et al. "Rather than being an oddity, NNMs are a normal step in the chemical bonding of homonuclear groups, if analyzed in the appropriate range of internuclear distances. For most elements, however, this range occurs far away from the stable geometry under normal thermodynamic conditions" (93)

(b) The interatomic surface: this is the locus of space where

$\nabla \rho(\mathbf{r}) \cdot \mathbf{n}(\mathbf{r})=0$

$\mathbf{n}(\mathbf{r})$ is a vector normal to the surface. ${ }^{17}$ An interatomic surface separates each atomic domain from the others and defines the volume of the atomic basin.

(c) The bond path: two atomic domains sharing an interatomic surface are necessarily connected by a line of maximal electron density which crosses the interatomic surface at the bond critical point, i.e. a saddle point with two negative and one positive curvatures of the electron density.

(d) The critical points: topological rules imply that saddles, maxima and minima be present in the electron density distribution. All these stationary points are characterized by three independent curvatures, with the same sign (like maxima and minima) or opposite signs (saddles). Therefore one can recognize the maxima (nuclear attractors),

17 The nuclear position must be excluded from this definition (either if the gradient is defined or not). 
the saddles (bond critical points or ring critical points) and the minima (cage critical points). Topological considerations lead to a precise correlation between the total number of all critical points, the so called Poincaré-Hopf relationship (94):

$\mathrm{N}_{\mathrm{c}}-\mathrm{N}_{\mathrm{r}}+\mathrm{N}_{\mathrm{b}}-\mathrm{N}_{\mathrm{n}}=\mathrm{c}$

where $N_{c}$ is the number of cage critical points, $N_{r}$ the number of ring critical points, $\mathrm{N}_{b}$ the number of bond critical points, $\mathrm{N}_{\mathrm{n}}$ the number of nuclear attractors and $\mathrm{c}$ is a constant ( $\mathrm{c}=-1$ for a finite system, $\mathrm{c}=0$ for an infinitely periodic system, taking into account all the critical points of a unit cell).

No matter how the electron density is defined, from a molecular orbital wave function, from a multipolar expansion or even from a discrete series of values on a grid, it is always possible to analyze it in topological terms, locating the critical points and defining the atomic domains.
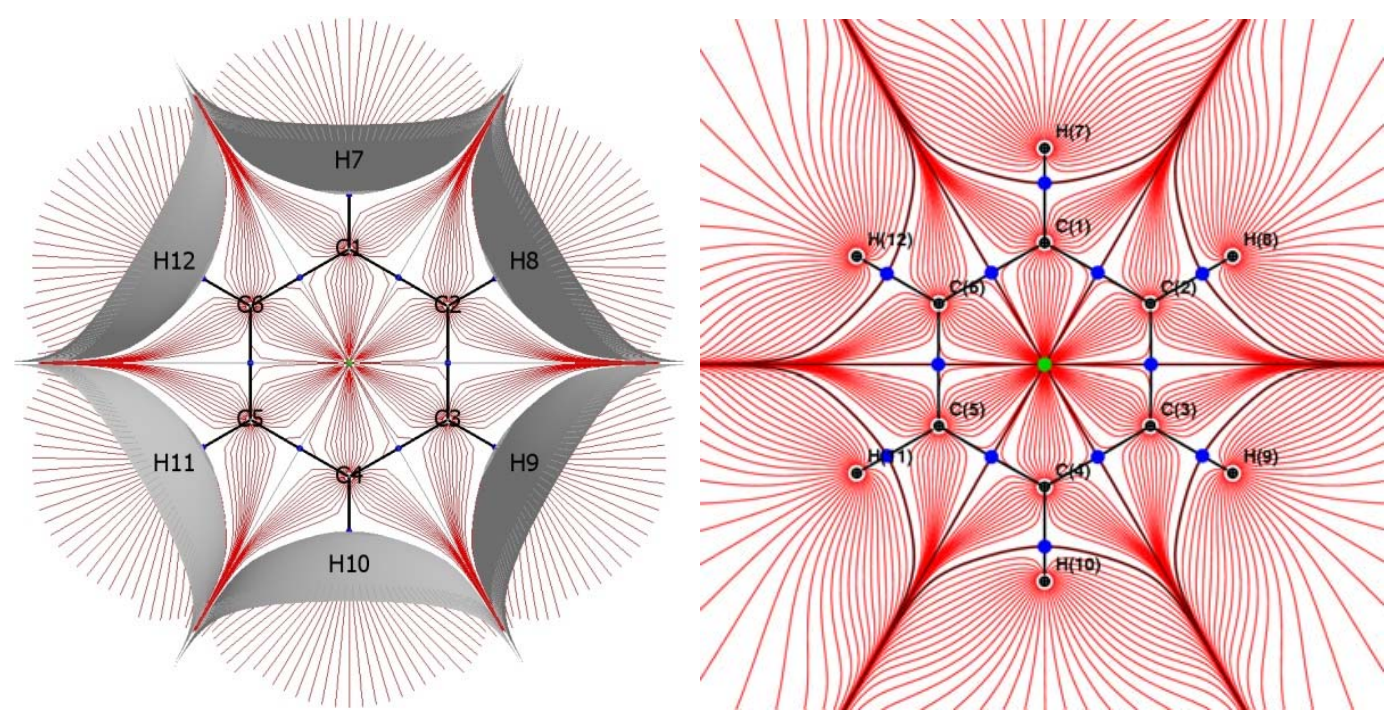

Figure 6 The QTAIM partitioning of molecular benzene from the theoretical wave function (left) or from the multipolar reconstruction (right). Gradient paths are shown for each atomic basin (red lines). Bond paths are black lines, bond critical points are 
blue spheres and the ring critical point is a green sphere. In the left figure the $\mathrm{C}-\mathrm{H}$ interatomic surfaces are also represented in grey.

As an example, in Figure 6, we see the density gradient partitioning of the benzene molecule, as calculated in section 2 from theoretical ab initio wave functions or from multipolar reconstruction of the same electron density through structure factor fitting. The red lines represent all the gradient trajectories terminating at the nuclear attractors. The grey surfaces are the interatomic surfaces. The solid black lines are the bond paths that form the molecular graph, an interesting representation of a molecule, which could correspond to a traditional visualization of the chemical bonds, but with some distinctions:

(a) The bond path does not correspond to a covalent two-center bonding, but it simply highlights the connection between two atoms.

(b) The bond path does not discriminate the total number of electrons shared between two atoms (ranging from 0 to all electrons belonging to the atoms).

(c) The bond path is not necessarily a straight line and could be significantly longer than the simple geometrical vector connecting the two atoms, especially for very strained systems (like cyclic molecules)

(d) The bond path cannot represent through bond or multi-center interactions, but it is obviously affected by them.

The actual meaning of the bond path is well described in a seminal paper by Bader (95), where some criticisms (96) associated with identifying the bond path with the chemical bond are discussed. 


\subsubsection{Chemical Bonding analysis}

It is noteworthy that an immense literature describes the characterization of the nature of the chemical bond, based on the analysis of the electron density distribution and its derivative at the bond critical points (97). The main conclusion of this enormous work was that it is basically impossible to provide a sensible and comprehensive classification of chemical bonds based only on a few quantities determined at the bond critical points, even if energy densities are also considered (i.e. functions that distribute the total, the kinetic and the potential, energy of a system per each unit of volume (82)). On the other hand it is now clear that the nature of the chemical bond may be revealed by a more comprehensive analysis, including:

(a) the calculation of integral properties of the atomic domains (atomic populations, atomic energies, mulipolar moments, etc.);

(b) the calculation of properties of the two electron density, for example electron delocalization indices (98) that measure the amount of covalent character in a bond by counting the fraction of electron pairs shared between two atomic basins.

(c) a breakdown of interatomic energy in terms that more precisely reflect the covalent or non-covalent bonding mechanism, for example by means of the so called interacting quantum atom approach (99), that defines all the bonding contributions per each atom using QTAIM partitioning.

While a topological analysis of the QTAIM kind is always feasible from an electron density distribution, some yet more sophisticated analyses require instead the two particle density. This rules out some of the refinement methods of the experimental 
electron density against measured X-ray diffraction intensities, in particular the multipolar model or the electron density on a grid as available from MEM. On the other hand, methods like the X-ray constrained wave function or the density matrix refinement could return some quantities otherwise available only from purely theoretical methods.

As discussed above, it has become clear that Bader's QTAIM topological analysis cannot alone reveal the nature of chemical bonding. The reason is simply explained by the nature of the density matrices introduced in equations [2-4]: the chemical bonding resides in the off-diagonal terms, whereas the electron density is the trace of the first order density matrix. Thus, $\rho(\mathbf{r})$ is determined by the chemical bonding but the nature of the chemical bonding is not entirely retrievable from $\rho(\mathbf{r})$ only. For this reason, the indicators based on the pair density (like the delocalization indices) are necessary for a full comprehension as well as the analyses of other functions based on pair density, like the electron localization function (100) or the electron localizability indicator (101), which are brilliantly illustrated in ref. (102). All these functions try to quantify and visualize the electron pairing occurring upon covalent bonding, which is "invisible" in the one electron density. The disadvantage of these functions is they are not available experimentally. For this reason, Gatti proposed the systematic use of the source function (103), an observable Green's influence function (104) of the one electron density:

$\rho(\mathbf{r})=\int \operatorname{LS}\left(\mathbf{r}, \mathbf{r}^{\prime}\right) \cdot d \mathbf{r}^{\prime}(23)$

Where LS is the local source:

$$
\operatorname{LS}\left(\mathbf{r}, \mathbf{r}^{\prime}\right)=-\left(4 \pi \cdot\left|\mathbf{r}-\mathbf{r}^{\prime}\right|\right)^{-1} \cdot \nabla^{2} \rho\left(\mathbf{r}^{\prime}\right)
$$


The applications of the source function are well described in ref. (105). In particular, the integral properties and the partitioning are extremely intriguing, as one may equate $\rho(\mathbf{r})$ to a sum of $\mathrm{S}(\mathbf{r} ; \Omega)$ (terms integrated over each atomic basic $\Omega$ ):

$\rho(\mathbf{r})=\mathrm{S}(\mathbf{r}, \Omega)+\sum_{\Omega^{\prime} \neq \Omega} \mathrm{S}\left(\mathbf{r}, \Omega^{\prime}\right)$

An analysis of the source function at the important topological loci is expected to provide information on the nature of chemical bonding, although this function is not at all connected with the pair density but only with the one electron density.

Unfortunately, the atomic terms in equation (25) have often been interpreted by other authors as a kind of population, like a charge or an orbital occupancy, which is an incorrect view given that $\mathrm{S}(\mathbf{r} ; \Omega)$ could be a source (positive) or a sink (negative) and it is better understood as the determination of the density, rather than a contribution to the electron density at $\mathbf{r}$ (in the same way as the water density at a given point is determined by the pressure at another point in an hydraulic system). This mistaken view has highly affected some interpretations.

On the other hand, Gatti stressed the ability of source functions to visualize the localization and delocalization of electrons and therefore their utility to analyse the nature of the chemical bonding. However, this view received some criticism (106) especially for the ability to really represent electron sharing and long range, throughbond, interactions.

\subsection{Hirshfeld analysis and conceptual DFT}

Following Bader's QTAIM, other partitioning schemes have been proposed, the most relevant being the Hirshfeld's approach (111), based on a very simple partitioning based on a kind of "financial approach" to the electron density: each atom is viewed as a 
stockholder that shares the profits or losses of the electron density distribution (more adequately the charge concentrations or depletions) in accordance with the "investment", that is the amount of electron density that a spherical atom would independently provide at each point. Thus, in a very simple way, the Hirshfeld atomic electron density is obtained by

$\rho_{i}(\boldsymbol{r})=\rho(\boldsymbol{r}) \frac{\rho_{i}^{I A M}(\boldsymbol{r})}{\sum_{j}^{N} \rho_{j}^{I A M}(\boldsymbol{r})}$

where $\rho_{i}(\boldsymbol{r})$ is the electron density at point $\mathbf{r}$ belonging to atom $\mathrm{i} ; \rho(\mathbf{r})$ is the total electron density of a molecule, $\rho_{i}^{I A M}(\boldsymbol{r})$ is the electron density calculated at point $\mathbf{r}$ from the spherical atom distribution of the atom $\mathrm{i}$ and $\sum_{\mathrm{j}}^{\mathrm{N}} \rho_{j}^{I A M}(\mathbf{r})$ is the total electron density obtained from the superposition of all the spherical atoms (also called as promolecular density). In Hirshfeld's definition, all atoms overlap as any atom contributes at least in a small part to the electron density at each point in space. ${ }^{18}$ This is the main difference with respect to QTAIM, which instead is a rigid partition of the molecular space. The other major difference is that Hirshfeld or stockholder partition is not quantum mechanical as the promolecule has no precise quantum mechanical meaning, despite some papers (107) which discuss it within a reappraisal of Berlin's theorem (108). Moreover, the promolecule is not energetically stable, since atoms with such a spherically distributed electron density would be more stable in isolation than bound within a molecule built from their superposition.

Besides these very important contra-indications, Hirshfeld's approach has found at least two very important applications: one is the atomic partitioning within the so called

\footnotetext{
${ }^{18}$ The electron density decays to zero at infinite distance from the nucleus.
} 
conceptual-DFT (109), where the stockholder scheme is used to retrieve atomic DFT functions; the other is the Hirshfeld's molecular partitioning proposed by Spackman and co-workers (110) with potential applications in crystal engineering (111), see Figure 7. Conceptual DFT provides a unified mathematical framework to correlate changes of the electron density with chemical reactivity and thermodynamics.

The approach suggested by Spackman, instead, represents an extension of Hirshfeld's idea to a crystal: as an atom in a molecule can be identified using equation [26], so a molecule in a crystal can be defined replacing the atomic weight with a molecular weight. However, Spackman recognized the necessity to define precise spatial regions for molecules, and therefore he proposed to assign to a molecule the volume that is owned (by a stockholder, to pursue the analogy above further) at least at $50 \%$ (that is $\frac{\rho_{i}^{I A M}(\boldsymbol{r})}{\sum_{j}^{N} \rho_{j}^{I A M}(\boldsymbol{r})} \geq 0.5$, where $i$ and $j$ here runs over all molecules in a crystal, instead of atoms). The volume is defined by a surface, the so-called Hirshfeld surface, which was found not only useful to illustrate the shape of a molecule in a crystal but also to plot other properties (like the lengths of intermolecular contacts) to characterize the binding properties of the molecule, a tool particularly useful in crystal engineering.

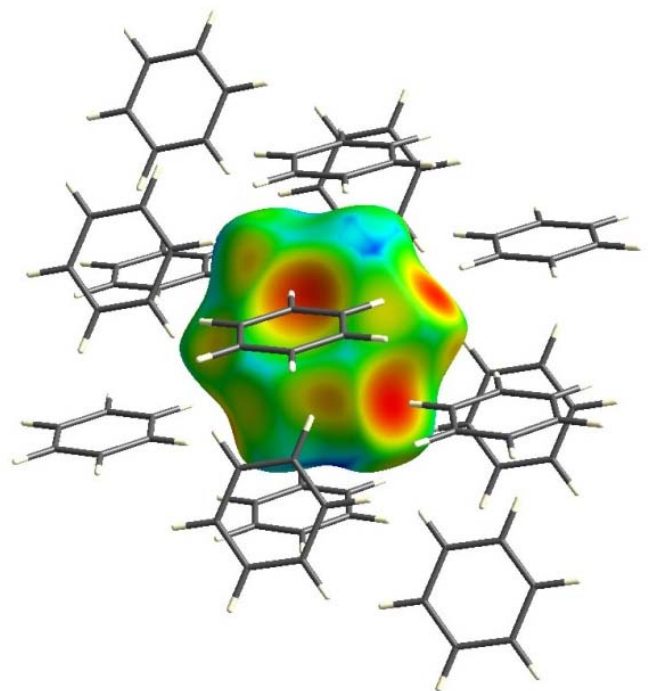


Figure 7. The Hirshfeld surface of a benzene molecule in the benzene P $21 / c$ crystal structure. The surface shown includes within it a benzene molecule. The stick representation molecules are the nearest neighbours to the Hirshfeld surface encapsulated benzene molecule. The color coding emphasizes closer intermolecular contacts (red), intermediate contacts (green) and very distant contacts (blue).

\subsection{Fine and coarse grain approaches for the intermolecular interactions}

The last kind of analysis which is shown in this Tutorial review is concerned with the quantification of binding attitudes of molecules in supramolecular aggregations.

Both materials science and pharmaceutical drug design require nowadays precise information on the distribution of electrons in the building blocks that constitute a material or a biomolecule, because this is fundamental to predict their functionalities and the abilities to aggregate, in a crystal or an agglomeration of molecules such as in a pharmaceutical pill containing a drug.

In fact, methods to anticipate binding energies between two or more molecular building blocks have been known for a long time, based on simple electrostatic charge schemes or more sophisticated descriptions. For example, the multipolar expansion is normally used to approximate the electrostatic potential of molecules (112) (see Figure 8) and allows one to calculate, at least approximately, the electrostatic interaction energies between two molecules (113). This approach has been widely adopted also for tentative predictions of solid state aggregation (the field of crystal structure prediction), a well known hot topic in modern structural science (114). 

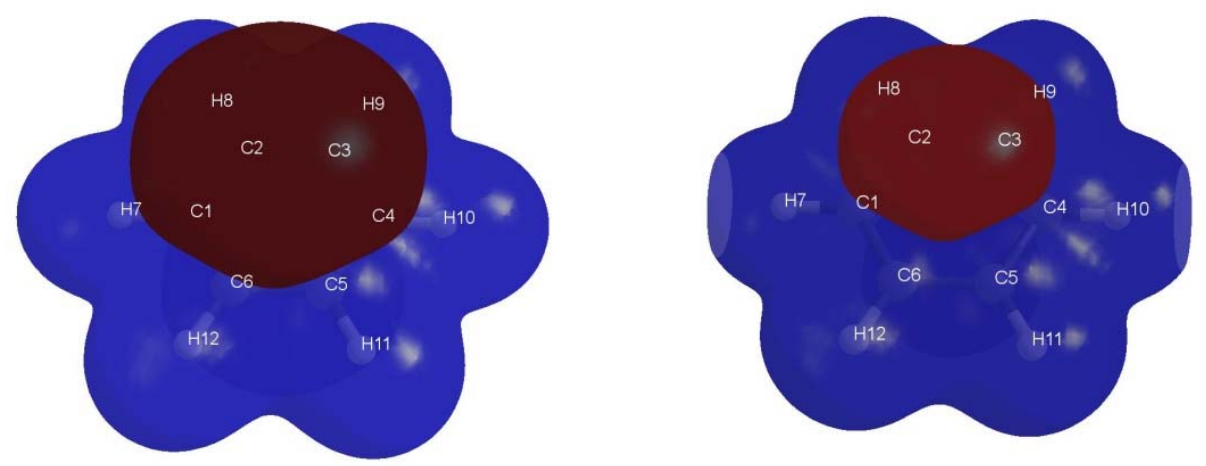

Figure 8 The Electrostatic potential, $\varphi(\mathbf{r})=\int \frac{\rho\left(\mathbf{r}^{\prime}\right)}{\left|\mathbf{r}-\mathbf{r}^{\prime}\right|} d \mathbf{r}^{\prime}$, of benzene from B3LYP/6$31 \mathrm{G}(2 \mathrm{~d}, 2 \mathrm{p})$ (left) and from multipolar reconstruction (right). Isosurfaces at $+0.025 \mathrm{e} \AA^{-1}$ (blue) and $-0.025 \mathrm{e}^{-1}$ (dark red).

In a more comprehensive treatment, one should consider the interaction energies not only in terms of pure electrostatics, but also including perturbations due to quantum mechanical phenomena. In fact, the interaction between two charge or multipolar distributions $A$ and $B$ could easily be described using Coulomb's Law or Buckingham (113) extension to higher multipoles, both based on classical electrostatics:

$$
\begin{aligned}
& E_{e s}(A B)=\sum_{a \in A b \in B} \sum_{A} T q_{a} T q_{b}+T_{\alpha}\left(q_{a} \mu_{\alpha, b}-q_{b} \mu_{\beta, a}\right)+ \\
& T_{\alpha \beta}\left(1 / 3 q_{a} \Theta_{\alpha \beta, b}+1 / 3 q_{b} \Theta_{\alpha \beta, a}-\mu_{\alpha, a} \mu_{\beta, b}-\mu_{\alpha, b} \mu_{\beta, a}\right)+\ldots .
\end{aligned}
$$

with implicit summations (following Einstein's convention) over Greek indices. The $a$ and $b$ indexes run over all atoms belonging to the $A$ or $B$ molecule, $\mu_{\alpha}$ and $\Theta_{\alpha \beta}$ are electric dipole and quadrupole moment components, whereas $T_{\alpha \beta \gamma \ldots \text { are the symmetrical }}$ interaction tensors $\left(\nabla_{\alpha} \nabla_{\beta} \nabla_{\gamma} \ldots(\mathbf{r a b})^{-1}, \mathbf{r a b}_{\text {ab }}\right.$ being the vector from the origin of "a" to "b". The sum extends up to the higher multipole-multipole interaction description and the 
summation generally converges for $l_{a}+l_{b}>5$ ( $l$ being the order of the multipolar expansion on center " $a$ " and " $b$ ", respectively).

The multipole approximation is quite correct at large intermolecular distances, but in the short range the mutual penetration of the two electron distributions creates a large perturbation. The actual space distribution of the two interacting electron densities should be properly considered. Spackman recognized this [115], but the model was lacking the most important part of the penetration as later recognized [116]. Volkov [117] and Gavezzotti [118] proposed volume integration of the electron densities for the evaluation of the exact electrical potential. A combination of exact-potential at short atom-atom distances and multipole model at large atom-atom distances [119] has now become the standard.

So far we have assumed that electrons are statically distributed, but in reality the observed electron density only describes the averaged probability to find electrons at a given point in space. This average comes from many possible electronic configurations and quantum states (in principle an infinite number), which have a given probability to occur and therefore they yield instantaneous deviations from the average distribution, creating instantaneous multipoles that are different from the static multipole distribution (that normally are refined against X-ray diffraction intensities or calculated from molecular wave functions). The instantaneous multipoles of two molecules produce an additional interaction between them, which gives rise to the so called London forces, more commonly known as van der Waals interactions. Moreover one should further consider that the electrons are Fermions and therefore cannot be described without taking into account the anti-symmetrization of their wave function, in order to obey the Pauli exclusion principle (8). 
The London forces produce a mutual, albeit small, stabilization between two molecules , also called the dispersion energy (and which are an order of magnitude smaller than a Coulomb interaction between two ions and in turn also much smaller than the interaction between two permanent dipoles). A precise calculation of these London forces and corresponding energies would require a sophisticated theoretical treatment, including a configuration interaction that for systems of more than one molecule become easily prohibitive computationally. However, research in this direction has proven that some approximate force fields could be constructed based on the type of the atoms involved and of course on the distance between them. For example the well known Lennard-Jones potentials (120) include an $\mathrm{r}^{-6}$ stabilizing term, and this coefficient tries to reproduce the dispersive attractive interaction between two molecules (A and B). Notably, the Coulomb electrostatic interaction energy decreases as a function of $\mathrm{r}^{-2}$ therefore the stabilizing dispersion energy is over a much shorter range than a charge-charge interaction and somewhat similar to a quadrupolequadrupole interaction (for example that occurring between two molecules of benzene). More sophisticated force fields include $\mathrm{r}^{-8}$ and $\mathrm{r}^{-10}$ terms, as well as anisotropic interactions (121), hence a tensorial form for the coefficients is needed. The more flexible force fields (i.e. those using more parameters) are based on distributed atomic interactions. Therefore for each pair of molecules all the atom-atom interactions are considered.

$$
E_{\text {disp }}(A B)=\sum_{i \in A} \sum_{j \in B} \frac{C_{a b}^{(6)}}{r_{a b}^{6}}+\frac{C_{i j}^{(8)}}{r_{a b}^{8}}+\frac{C_{i j}^{(10)}}{r_{a b}^{10}}+\ldots
$$

The Fermions' behavior produces a local short range destabilization (normally called 
Pauli repulsion). ${ }^{19}$ The potential associated with this destabilization has been described in forms of a term varying as $\mathrm{r}^{-12}$

$$
E_{\text {rep }}(A B)=\sum_{i \in A} \sum_{j \in B} \frac{\sigma_{a b}}{r_{a b}^{12}}
$$

or expressed as an exponential function

$$
E_{\text {rep }}(A B)=b_{A} b_{B} \exp \left[\left(c_{A}+c_{B}\right) r\right]
$$

In both equations (28) and (29) it is evident that this term is quite short range and it becomes dominant at very short distances. In fact, two electron density distributions tend to avoid each other mainly because of the Pauli principle at very short range or because of electrostatic forces (at a medium-large range). Nuclear charges (which also repulse each other because of Coulomb forces) could then compensate the electronelectron destabilization producing a larger electrostatic attraction (nucleus to electrons). The overall stabilization or destabilization depends on the overall amount and distribution of positive and negative charges.

Two additional terms are necessary to fully understand the interaction between two molecules: one is the so-called induction (or polarization) energy, the other is an account of covalent effects, sometime reduced to as a charge transfer term (that cannot be taken however as a classical electrostatic term).

${ }^{19}$ Of course, there is no Pauli force in quantum mechanics. However, the Fermi Hole energy, due to the Pauli exclusion principle, can be associated with a potential (that is normally used in semi-empirical force fields) whose gradient generates an apparent force, which is necessarily repulsive for each pair of atoms. 
The induction energy is the stabilization obtained by mutual polarization of the molecule by another and it depends on the electric field of one molecule and the polarizability of the other (and vice versa). Noteworthy, however, if a molecular electron density is refined against single crystal X-ray diffraction data, or otherwise calculated in periodic systems, then the electrostatic energy and the induction energy are basically merged and indistinguishable, because the electron density distributions are already including the effects of such mutual polarizations (122). It is important to note that while the induction energy is the interaction between the static field produced by one molecule and the polarizability of the other, the dispersion energy (in a first order approximation) comes from the interaction between the two molecular charge distributions, given the well known relationship also proposed by London (123):

$$
E_{\text {dispersion }}(A B)=-\frac{3}{2} \frac{E_{A}^{i o n} E_{B}^{i o n} \alpha_{A} \alpha_{B}}{\left(E_{A}^{i o n}+E_{B}^{i o n}\right) r_{A B}^{6}}
$$

where $E^{i o n}$ is the first ionization energy of a molecule and $\alpha$ is the molecular polarizability.

The covalent effects that we anticipated above may play an important role for very short range interactions, like strong and very strong hydrogen bonds, where the covalent mechanism is dominating. In fact here the assumption made above, that a short range potential is entirely due to the anti-symmetrization of the relative wave functions of the two molecules, is no longer valid and an analogous, stabilizing, exchange potential should be considered. For example, very strong hydrogen bonds are better simulated including at least empirical force fields mimicking the covalent interactions (124). Espinosa and co-workers (125) proposed energy density indicators for the evaluation of the strength of hydrogen bonds $\mathrm{X}-\mathrm{H}---\mathrm{Y}$, in particular correlating the potential energy 
density at the $\mathrm{H}---\mathrm{Y}$ bond critical point with the dissociation energy of the aggregate:

$$
E(A B)=\frac{1}{2} V_{b c p}
$$

This correlation was obtained from a series of experimentally calculated topologies. There are some remarks to consider, however. The energy density at the bond critical point is not accounting for the overall intermolecular interactions, in particular when charged molecules are concerned. The most relevant example is the strong hydrogen bond between two ions (126), that normally is not sufficient to guarantee an overall stability of the aggregate, for which counter-ions are also necessary. Nevertheless, the energy density would address the stabilization produced by the hydrogen bond, namely the local attraction in the frame of a global repulsion. It is also interesting that an energy density is measured in units of $\mathrm{e}^{2} \AA^{-4}$, that is a pressure. Therefore the $1 / 2$ coefficient in equation (31) is actually not dimensionless. Recently, the kinetic energy density at the bond critical points of the weaker intermolecular interactions has instead been adopted to correlate with the bulk modulus of the crystal (127).

We conclude this section by mentioning the potential application of electron density multipolar expansion to calculate optical or mechanical properties of crystals: the atomic/molecular multipoles can be used to calculate crystal elastic constants (128) or crystal susceptivities (129), through Ewald summation (130). This has stimulated some interesting studies on linear and non-linear optical properties of molecular crystals (131), using the X-ray constrained wave function method.

In Table 3, the main crystal or molecular properties available from various electron density models are summarized. In particular, the limitations of each method are clearly outlined. 
Table 3 Possible analyses of electron density derived properties from the different models. Note that this table does not take into account the actual availability of these functions in the commonly adopted software packages.

\begin{tabular}{|c|c|c|c|c|c|c|c|c|c|}
\hline$\rho(\mathbf{r})$ & $\begin{array}{c}\text { Deformation } \\
\text { densities }\end{array}$ & $\begin{array}{l}\text { QTAIM } \\
\text { topological } \\
\text { analysis }\end{array}$ & $\begin{array}{l}\text { Hirshfeld } \\
\text { multipoles }\end{array}$ & $\begin{array}{l}\text { Delocalization } \\
\text { indexes and } \\
\text { pair density }\end{array}$ & $\begin{array}{c}\text { Source } \\
\text { Function and } \\
\text { Information } \\
\text { theory }\end{array}$ & $\begin{array}{c}\text { Intermolecular } \\
\text { Electrostatic } \\
\text { interaction }\end{array}$ & $\begin{array}{c}\text { Total } \\
\text { intermolecular } \\
\text { Interactions }\end{array}$ & $\begin{array}{c}\text { Crystal } \\
\text { Elastic } \\
\text { properties }\end{array}$ & $\begin{array}{c}\text { Crystal } \\
\text { Optical } \\
\text { properties }\end{array}$ \\
\hline $\begin{array}{c}\text { Multipolar } \\
\text { expansion }\end{array}$ & $\mathrm{Y}$ & $\mathrm{Y}$ & $\mathrm{Y}$ & N.A. & $\mathrm{Y}$ & $\begin{array}{l}\text { induction energy } \\
\text { implicit }\end{array}$ & Only empirically & $\begin{array}{c}\text { From } \\
\text { multipole } \\
\text { summation }\end{array}$ & $\begin{array}{c}\text { semi- } \\
\text { empirical } \\
\text { based on } \\
\text { multipoles }\end{array}$ \\
\hline MEM & $\begin{array}{c}\mathrm{Y} \text { (only } \\
\text { dynamic) }\end{array}$ & $\begin{array}{c}\text { only } \\
\text { numerical, } \\
\text { thermal } \\
\text { average } \\
\text { density }\end{array}$ & $\begin{array}{l}\text { thermal } \\
\text { average } \\
\text { density }\end{array}$ & N.A. & $\begin{array}{c}\text { only } \\
\text { numerical, } \\
\text { thermal } \\
\text { average } \\
\text { density }\end{array}$ & $\begin{array}{l}\text { induction energy } \\
\text { implicit; } \\
\text { multipoles from } \\
\text { numerical } \\
\text { integration; } \\
\text { thermal average } \\
\text { density }\end{array}$ & Only empirically & $\begin{array}{c}\text { From } \\
\text { multipole } \\
\text { summation }\end{array}$ & $\begin{array}{l}\text { semi- } \\
\text { empirical } \\
\text { based on } \\
\text { multipoles }\end{array}$ \\
\hline $\begin{array}{c}\text { X-ray } \\
\text { constrained } \\
\text { wave function }\end{array}$ & $\mathrm{Y}$ & $\begin{array}{c}\text { only } \\
\text { molecular }\end{array}$ & $\mathrm{Y}$ & $\begin{array}{l}\text { only molecular } \\
\text { and } \\
\text { approximated }^{(a)}\end{array}$ & $\mathrm{Y}$ & $\begin{array}{l}\text { induction energy } \\
\text { implicit }\end{array}$ & $\mathrm{Y}$ & $\begin{array}{c}\text { From } \\
\text { multipole } \\
\text { summation } \\
\end{array}$ & $\begin{array}{c}\text { From } \\
\text { Lorentz } \\
\text { summation } \\
\end{array}$ \\
\hline $\begin{array}{l}\text { Molecular } \\
\text { orbital Wave } \\
\text { function }\end{array}$ & $\mathrm{Y}$ & $\begin{array}{c}\text { only } \\
\text { molecular }\end{array}$ & $\mathrm{Y}$ & $\begin{array}{l}\text { only molecular, } \\
\text { approximated for } \\
\text { DFT wave } \\
\text { functions } \\
\text { (b) }\end{array}$ & $\mathrm{Y}$ & $\mathrm{Y}$ & $\mathrm{Y}$ & $\begin{array}{c}\text { From } \\
\text { multipole } \\
\text { summation }\end{array}$ & $\begin{array}{c}\text { From } \\
\text { Lorentz } \\
\text { summation }\end{array}$ \\
\hline $\begin{array}{c}\text { HF or DFT } \\
\text { Crystal orbital } \\
\text { wave function }\end{array}$ & $\mathrm{Y}$ & $\mathrm{Y}$ & $\mathrm{Y}$ & $\begin{array}{c}\text { Exact at HF } \\
\text { level, } \\
\text { approximated at } \\
\text { DFT }^{(b)}\end{array}$ & $\mathrm{Y}$ & $\begin{array}{l}\text { induction energy } \\
\text { implicit }\end{array}$ & $\mathrm{Y}$ & $\mathrm{Y}$ & $\mathrm{Y}$ \\
\hline $\begin{array}{l}\text { DFT Crystal } \\
\text { Plane waves }\end{array}$ & $\mathrm{Y}$ & $\mathrm{Y}$ & $\mathrm{Y}$ & approximated $^{(\mathrm{b})}$ & $\mathrm{Y}$ & $\begin{array}{c}\text { induction energy } \\
\text { implicit }\end{array}$ & $\mathrm{Y}$ & $\mathrm{Y}$ & $\mathrm{Y}$ \\
\hline
\end{tabular}

(a) The definition of the correlation density in these wave functions is not defined; (b) At DFT level the pair density is not defined, but an approximated function can be constructed using the Kohn-Sham orbitals in a similar way as the HF wave function (132). 


\section{Conclusions and outlook}

This Tutorial review has presented the basics of the topic, including the motivations and the methodologies for modeling electron density in molecules and solids from quantum mechanical methods or from X-ray diffraction data. It also focused on the expectations and limitations of the various approaches.

It was shown that the amount of quantum mechanical prior information necessary for the modeling from experimental data is nowadays very large and the so-called classical challenge has now been inverted: theory is more and more challenging the experiments and only the most accurate measures can reveal features anticipated by first principle calculations.

This is mainly due to the more limited progress made on the experimental side: in fact, despite large technological improvement (X-ray sources, detectors etc.), the problem is that it is still elastic X-ray diffraction that is the most adopted method for electron density determination. New approaches, however, have been recently proposed, for example the combined X-ray, polarized neutron and Compton X-ray scattering that can allow one to simultaneously refine charge, spin and momentum densities derived from experiments (51) enlarging the traditional point of view of an electron density determination.

The framework of the field might also change thanks to the introduction of free electron X-ray lasers, because the electron density of excited states might be more accurately measured, challenging the theoretical interpretation. 


\section{Acknowledgements}

This Tutorial review is dedicated to the late Department of Structural Chemistry and Inorganic Stereochemistry of the University of Milan, where I had occasion to perform many studies before moving to the University of Bern. The referees are thanked for very useful comments and remarks. The Swiss National Science Foundation (Project Nr. 141271 ) is thanked for financial support.

\section{Subject index}

atomic basin (atomic domain)

anharmonic motion

bond critical point

bond path

Bragg scattering

cage critical point

core refinement

Compton scattering

Coulomb force

critical point

deformation density

delocalization index

Density functional theory (DFT)

density matrix

Dirac-Fock equation

dispersion energy

Electrostatic potential (electric potential)

Fourier transform (FT)

Gaussian type functions

gradient path

Gram Charlier expansion

Green's influence function

Hansen-Coppens model

Hartree-Fock

energy

equation

wave function

Hellmann-Feynman theorem

Hirshfeld surface

hydrogen bond

independent atom model (IAM)

induction energy

interatomic surface

Lennard-Jones potential

local coordinate system

London forces

Maximum Entropy Method (MEM)

molecular graph

multipolar model

neutron diffraction

non-nuclear maxima (NNM)

one electron density

Pauli Exclusion Principle

Poincaré-Hopf relationship

polarized neutrons (PN)

promolecule

Quantum theory of atoms in molecules (QTAIM) 
Radial functions

residual index

ring critical point

Roothaan equation

Schrödinger equation

Schwinger's quantum action principle

Slater Type Orbital (STO)

source function (SF)

spherical harmonics

spin density

Stewart's atom

stockholder partitioning

suitability indicator

thermally averaged electron density

topological analysis

two electron density (pair density)

$\mathrm{X}$-ray constrained wave functions

$\mathrm{X}$-ray structure factors

zero point vibration

\section{Author's note:}

Since 2009, Dr Piero Macchi has led the chemical crystallography group at the Department of Chemistry of the University of Bern, Switzerland. His research is in the field of accurate crystallography under non-ambient conditions in order to reveal chemical bonding features.

This research involves electron density analysis and high pressure crystallography on materials interesting for their chemical and physical properties, in particular metal complexes, metal organic frameworks and optoelectronic organic materials. His $\mathrm{PhD}$ is in Chemistry and was awarded by the University of Milano, Italy in 1999. He is a member of the IUCr Commission on Charge, Spin and Momentum Densities, Chair of the special interest group on Charge, Spin and Momentum density of the European Crystallographic Association, Treasurer and Vice President of the Swiss Society for Crystallography and Chairman of the Gordon research Conference on Electron Density Distribution and Chemical Bonding.

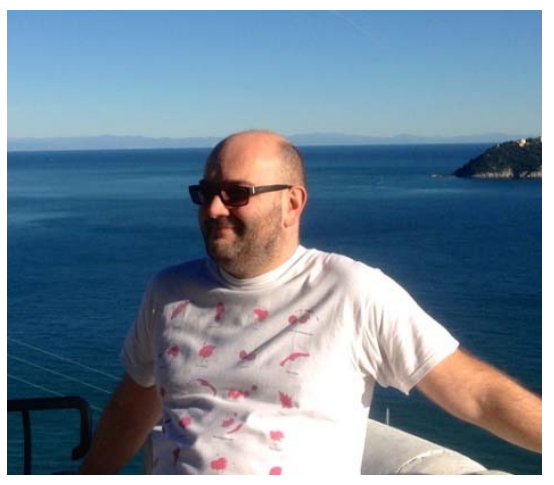




\section{References}

[1] Debye, P. X-ray dispersal. Ann. Phys.1915, 46, 809-823.

[2] R.W. James X-Ray Crystallography Methuen \& Co.Ltd. 1961

[3] Coppens, P. Comparative X-ray and Neutron diffraction study of bonding effects in s-triazine. Science. 1967, 158, 15771579.

[4] a) Hohenberg, P.; Kohn, W. Inhomogeneous electron gas. Phys. Rev. 1964, 136, 864-871 ; b) Kohn,W.; Sham, L.J. SelfConsistent Equations Including Exchange and Correlation Effects. Phys. Rev. 1965, A 140, 1133-1138; c) Parr R.G., Yang, W. Density-Functional Theory of Atoms and Molecules. International Series of Monographs on Chemistry 16, Oxford Science Publications, Oxford (1989)

[5] (a) Heisenberg, W. On the Perceptual Content of Quantum Theoretical Kinematics and Mechanics. Z. Physik. 1927, 43 172-198; (b) English Translation by Wheeler, J. A.; Zurek, W. eds. Quantum Theory and Measurement. Princeton: Princeton University Press, 1983: 62-84.

[6] McWeeny, R. Methods of Molecular Quantum Mechanics (second edition). Academic Press, London, 1989.

[7] Born, M.; Oppenheimer, R. Zur Quantentheorie der Molekeln. Annalen der Physik 1927, 389, 457-484.

[8] Pauli, W. On the connection of the arrangement of electron groups in atoms with the complex structure of spectra. $Z$. Phys.1925, 31, 765-783.

[9] a) Gatti, C.; Macchi, P. (editors) Modern charge density analysis, Springer, 2012; b) Deutsch, M.; Claiser, N.; Pillet, S.; Chumakov, Y.; Becker, P. ; Gillet, J.-M.; Gillon, B.; Lecomte, C.; Souhassou M. Experimental determination of spindependent electron density by joint refinement of X-ray and polarized neutron diffraction data. Acta Cryst. 2012, A68, 675-686.

[10] Chimpri, A. S.; Gryl, M.; Dos Santos L. H. R.; Krawczuk, A.; Macchi, P. Correlation between accurate Electron density and Linear Optical Properties in Amino acid derivatives. 1. L-Histidinium Hydrogen Oxalate. Submitted.

[11] Meindl, K.; Henn, J. Foundations of residual-density analysis. Acta Cryst. 2008, A64, 404-418.

[12] Jørgensen, M. R. V.; Svendsen, H.; Schmøkel, M. S.; Overgaard, J. ; Iversen, B. B. On the significance of Bragg reflections Acta Cryst. 2012, A68, 301-303.

[13] Poulsen, R. D.; Bentien, A.; Graber, T.; Iversen, B. B. Synchrotron charge-density studies in materials chemistry: 16 K $\mathrm{X}$-ray charge density of a new magnetic metal-organic framework material, $\left[\mathrm{Mn}_{2}\left(\mathrm{C}_{8} \mathrm{H}_{4} \mathrm{O}_{4}\right)_{2}\left(\mathrm{C}_{3} \mathrm{H}_{7} \mathrm{NO}\right)_{2}\right]$. Acta Cryst. 2004, A60I, 382-389

[14] Stevens, E. D.; Coppens, P. A priori estimates of the errors in experimental electron densities Acta Cryst. 1976, A 32, 915-917.

[15] Farrugia, L. J.; Evans C. Experimental X-ray Charge Density Studies on the Binary Carbonyls $\mathrm{Cr}(\mathrm{CO})_{6}, \mathrm{Fe}(\mathrm{CO})_{5}$, and $\mathrm{Ni}(\mathrm{CO})_{4}$. J. Phys. Chem. 2005, 109, 8834-8848.

[16] Kato, T. On the eigenfunctions of many-particle systems in quantum mechanics Pure Appl. Math. 1957, 10, 151-177.

[17] Roothaan, C. C. J. New Developments in Molecular Orbital Theory. Reviews of Modern Physics 1951, 23, 69-89.

[18] Clementi, E.; Roetti, C.: Tables of Roothaan-Hartree-Fock Wavefunctions, Special Issue in Atomic Data and Nuclear Data Table, Academic Press, New York, 1974

[19] Clementi, E.; Raimondi, D. L. Atomic Screening Constants from SCF Functions. J. Chem. Phys 1963, 38, 2686-2689.

[20] a) Hellmann, H. Einfuhrung in die Quantenchemie, Deuieke, Leipzig, 1937; b) Feynman, R. P. Forces in Molecules. Phys. Rev. 1939, 56, 340-343.

[21] Born, M., Huang, K. Dynamical theory of Crystal lattices. Oxford, Clarendon Press, 1954.

[22] Willis, B.T.M., Pryor, A.W. Thermal vibrations in crystallography. Cambridge University Press, 1975.

[23] Madsen A. Ø. Modelling and analysing thermal motion in experimental charge density studies. in Gatti, C.; Macchi, P. editors Modern charge density analysis. Springer. 2012.

[24] Johnson, C. K. Addition of higher cumulants to the crystallographic structure-factor equation: a generalized treatment for thermal-motion effects. Acta Cryst. 1969, A25, 187-194.

[25] Trueblood, K.N.; Bürgi, H.B.; Burzlaff, H.; Dunitz, J.D.; Gramaccioli, C.M.; Schulz, H.H.; Shmueli, U.; Abrahams, S.C. Atomic Dispacement Parameter Nomenclature. Report of a Subcommittee on Atomic Displacement Parameter Nomenclature. Acta Cryst. 1996, A52, 770-781.

[26] Kuhs, W.F. Generalized atomic displacements in crystallographic structure analysis. Acta Cryst. 1992 A48, 80-98.

[27] Stewart, R. F. Generalized X-ray scattering factors. J. Chem. Phys. 1969, 51, 4569-4578.

[28] Gilbert, A. T. B.; Lee, A. M.; Gill. P. M. W. Methods for constructing Stewart atoms. J. Mol. Struct. (Theochem) 2000, 500, 363-374.

[29] a) Stewart, R. F.; Bentley, J.; Goodman, B. Generalized X-ray scattering factors in diatomic molecules. J. Chem. Phys. 1975, 63, 3786-3793; b) Stewart, R. F. Electron population analysis with rigid pseudoatoms. Acta Cryst. 1976, A32, $565-574$; c) Stewart, R. F. Electron population analysis with generalized x-ray-scattering factors - higher multipoles. $J$. Chem. Phys., 1973, 58, 1668-1676.

[30] a) Kurki-Suonio, K.; Meisalo V. Nonspherical deformations of the ions in Fluorite. J. Phys. Soc. Japan . 1966, 21, 122 126; b) Kurki-Suonio, K.; Meisalo V. Spherical harmonic expansions in X-ray diffraction analysis Ann. Acad. Sci. Fenn. 1967, A VI 241,18; c) Kurki-Suonio, K. On the information about deformations of the atoms in X-ray diffraction data. Acta Cryst. 1968, A24, 379-390; d) Kurki-Suonio, K. Site-symmetrized expressions of structure amplitudes. Acta Cryst. 1970, A26, 458-459; e) Kurki-Suonio, K. Symmetry and its implications. Isr. J. Chem. 1977, 16, 115-123; f) Kurki-Suonio, K. Charge deformation models. Isr. J. Chem. 1977, 16, 132-136.

[31] Coppens, P. X-Ray Charge Densities and Chemical Bonding. IUCr Texts on Crystallography 4. International Union of Crystallography, Oxford University Press, Oxford, 1997. 
[32] Hansen, N. K.; Coppens, P. Electron Population Analysis Of Accurate Diffraction Data. 6. Testing Aspherical Atom Refinements On Small-Molecule Data Sets. Acta Cryst. 1978, A34, 909-921.

[33] a) Hirshfeld, F. L. Space partitioning of the charge density. Isr. J. Chem. 1977, 16, 198-201; b) Hirshfeld, F. L. Deformation Density refinement program. Isr. J. Chem. 1977, 16, 226-229; c) Hirshfeld, F. L. Bonded-atom fragments for describing molecular charge-densities. Theor. Chim. Acta. 197, 44, 129-138; d) Hirshfeld, F. L. Can X-ray data distinguish bonding effects from vibrational smearing? Acta Cryst. 1976, A32, 239-244; e) Harel, M.; Hecht, M.; Hirshfeld, F. L. Electron-density refinements with an extended charge deformation model Acta Cryst. 1976, A31, S224;

[34] Spackman, M. A. Molecular Electric Moments from X-ray Diffraction Data. Chem. Rev. 1992, 92, 1769-1797

[35] Volkov, A., Macchi, P., Farrugia, L. J., Gatti, C., Mallinson, P., Richter, T., Koritsanszky, T. XD2006 - A Computer Program Package for Multipole Refinement, Topological Analysis of Charge Densities and Evaluation of Intermolecular Energies from Experimental and Theoretical Structure Factors 2006. Noteworthy, official versions of XD before 2003 contained sever errors that have seriously affected some studies.

[36] Stewart, R. F.; Spackman, M. A.; Flensburg, C. VALRAY - User's Manual, 2.1 ed. Carnegie Mellon University, Pittsburgh, PA, USA, and University of Copenhagen, Denmark 2000.

[37] For this program, written by N. K. Hansen, the official reference is ref (32).

[38] Jelsch C.; Guillot B.; Lagoutte, L.; Lecomte C. Advances in Proteins and Small Molecules. Charge Density Refinement Methods using software MoPro. J. Appl. Cryst. 2005, 38, 38-54.

[39] Petricek, V.; Dusek, M.; Palatinus, L. JANA2006, Structure Determination Software Programs. Institute of Physics, Praha. 2006.

[40] a) Pichon Pesme, V.; Lecomte, C.; Lachekar, H. On Building A Data-Bank Of Transferable Experimental ElectronDensity Parameters - Application To Polypeptides J. Phys. Chem..1995, 99, 6242-6250; b) Dittrich, B; Hubschle, C. B.; Luger, P; Spackman, M.A. Introduction and validation of an invariom database for amino-acid, peptide and protein molecules. Acta Cryst 2006, A62, 1325-1335.

[41] Kuhs, W. F. Generalized atomic displacements in crystallographic structure analysis. Acta Cryst. 1992, A48, 80-98.

[42] a) Becker, P. J.; Coppens, P. Extinction within the limit of validity of the Darwin transfer equations. I. General formalism for primary and secondary extinction and their applications to spherical crystals. Acta Cryst. 1974, A30, 129147; b) Becker, P. J.; Coppens, P. Extinction within the limit of validity of the Darwin transfer equations. II. Refinement of extinction in spherical crystals of SrF2 and LiF. Acta Cryst. 1974, A30, 148-153; c) Becker, P. J.; Coppens, P. Extinction within the limit of validity of the Darwin transfer equations. III. Non-spherical crystals and anisotropy of extinction. Acta Cryst. 1975, A31, 417-425.

[43] Clementi, E.; Raimondi, D. L. Atomic Screening Constants from SCF Functions. J. Chem. Phys., 1963, 38, 2686-2689.

[44] a) Su, Z.; Coppens, P. Nonlinear Least-Squares Fitting of Numerical Relativistic Atomic Wave Functions by a Linear Combination of Slater-Type Functions for Atoms with $\mathrm{Z}=1$-36. Acta Cryst., 1998, A54, 646-652; b) Macchi, P.; Coppens, P: Relativistic analytical wave functions and scattering factors for neutral atoms beyond $\mathrm{Kr}$ and for all chemically important ions up to I. Acta Cryst., 2001, A 57, 656-662; c) Volkov, A.; Macchi, P. unpublished (included in the software XD2006).

[45] Stone, A. J. Distributed multipole analysis, or how to describe a molecular charge-distribution. Chem. Phys. Lett. 1981, 83, 233-239.

[46] Stone, A. J. Distributed Polarizabilities. Mol. Phys. 1985, 56, 1065-1082.

[47] Roversi, P.; Barzaghi, M.; Merati, F.; Destro, R. Charge density in crystalline citrinin from X-ray diffraction at 19 K. Can. J. Chem., 1996, 74, 1145-1161.

[48] a) Becke, A. D. Density-Functional Thermochemistry. III. The Role of Exact Exchange J. Chem. Phys. 1993, 98, 56485652; b) Lee, C.; Yang, W.; Parr, R. G. Development of the Colle-Salvetti conelation energy formula into a functional of the electron density. Phys. Rev. B 1988, 37, 785-789.

[49] Figgis, B. N. Ligand Field Theory and Its Applications, Wiley-VCH, 2000.

[50] Holladay, A.; Leung, P. C.; Coppens, P. Generalized Relations Between d-Orbital Occupancies of Transition-Metal Atoms and Electron-Density Multipole Population Parameters from X-ray Diffraction Data. Acta Cryst., 1983, A39, $377-$ 387.

[51] a) Gillet, J. M.; Koritsanszky, T. Past, Present and Future of Charge Density and Density Matrix Refinements. in Gatti, C.; Macchi, P. editors Modern charge density analysis. Springer. 2012; b) Koritsanszky, T.; Volkov, A.; Chodkiewicz, M. New Directions in Pseudoatom-Based X-Ray Charge Density Analysis. in Stalke, D. Electron Density and Chemical Bonding, special issue of Structure and Bonding. 2012, 146, 1-26.

[52] Fischer, A.; Tiana, D.; Scherer, W.; Batke, K.; Eickerling, G.; Svendsen, H.; Bindus, N.; Iversen, B. B. Experimental and Theoretical Charge Density Studies at Subatomic Resolution. J. Phys. Chem. A. 2011, 115, 13061-13071.

[53] Jayatilaka, D.; Grimwood, D.J. Wavefunctions derived from experiment. I. Motivation and theory. Acta Cryst. A 2001, $57,76-86$

[54] Collins, D. M. Electron density images from imperfect data by iterative entropy maximization. Nature, 1982, 298, 4951 .

[55] Gull, S. F., Daniell, G. J.: Image Reconstruction from Incomplete and Noisy Data. Nature 1978, 272, 686-690.

[56] Jaynes, E. T. Prior probabilities. IEEE Trans. Syst. Sci. Cybern.1968, SSC-4, 227-240.

[57] Roversi, P.; Irwin, J. J.; Bricogne, G. Accurate charge-density studies as an extension of Bayesian crystal structure determination. Acta Cryst. 1998, A54, 971-996

[58] a) Palatinus, L., van Smaalen, S.: The prior-derived F constraints in the maximum-entropy method. Acta Cryst. A 61, 363-372 (2005); b) van Smaalen, S., Netzel, J.: The maximum entropy method in accurate charge-density studies. Physica Scripta, 79, 048304 (2009) 
[59] a) Hofmann, A.; Netzel, J.; van Smaalen, S. Accurate charge density of trialanine: a comparison of the multipole formalism and the maximum entropy method (MEM). Acta Cryst. 2007, B63, 285-295; b) Netzel, J.; van Smaalen, S. Topological properties of hydrogen bonds and covalent bonds from charge densities obtained by the maximum entropy method (MEM). Acta Cryst. 2009, B65, 624-638.

[60] van Smaalen, S.; Palatinus, L.; Schneider, M. The maximum-entropy method in superspace. Acta Cryst. 2003, A59, 459-469

[61] Palatinus, L.; Prathapa, S. J.; van Smaalen, S. EDMA: a computer program for topological analysis of discrete electron densities J. Appl. Cryst. 2012, 45, 575-580

[62] Katan, K.; Rabiller, P.; Lecomte, C.; Guezo, M.; Oisona, V.; Souhassou, M. Numerical computation of critical properties and atomic basins from three-dimensional grid electron densities. J. Appl. Cryst. 2003, 36, 65-73.

[63] Henkelman, G.; Arnaldsson, A.; Jonsson, H. A fast and robust algorithm for Bader decomposition of charge density. Comput. Mater. Sci. 2006, 36, 354-360.

[64] Bindzus, N.; Iversen, B. B. Maximum-entropy-method charge densities based on structure-factor extraction with the commonly used Rietveld refinement programs GSAS, FullProf and Jana2006 Acta Cryst. 2012, A68, 750-762.

[65] De Vries, R. Y.; Briels, W. J.; Feil, D. Critical analysis of non-nuclear electron-density maxima and the maximum entropy method. Phys. Rev. Lett. 1996, 77, 1719-1722.

[66] Stewart, R. F.; Davidson, E. R.; Simpson, W. T. Coherent X-Ray Scattering for the Hydrogen Atom in the Hydrogen Molecule. J. Chem. Phys. 1965, 42, 3175-3177.

[67] Roversi, P.; Destro, R. Approximate anisotropic displacement parameters for H atoms in molecular crystals. Chem. Phys. Lett. 2004, 386, 472-478.

[68] Tosoni, S.; Pascale, F.; Ugliengo, P.; Orlando, R.; Saunders V.R.; Dovesi, R. Vibrational spectrum of brucite, $\mathrm{Mg}(\mathrm{OH})_{2}$ : a periodic ab initio quantum mechanical calculation including $\mathrm{OH}$ anharmonicity. Chem. Phys. Lett. 2004, 396, 308-315.

[69] Gillon, B.; Becker, P. Magnetization Densities in Material Science. in Gatti, C.; Macchi, P. editors Modern charge density analysis. Springer. 2012.

[70] Schrödinger, E. An Undulatory Theory of the Mechanics of Atoms and Molecules. Phys. Rev. 1926, 28, 1049-1070.

[71] C. J. Cramer. Essential of Computational Chemistry. Theory and Models. Second Edition. John Wiley \& Son, Chichester, 2004.

[72] Gatti, C.; MacDougall, P. J.; Bader, R. W. F. Effect of electron correlation on the topological properties of molecular charge-distributions. J. Chem. Phys. 1987, 88, 3792-3804.

[73] Yanai, T.; Tew, D. P.; Nicholas, N. C. A new hybrid exchange-correlation functional using the Coulomb-attenuating method (CAM-B3LYP). Chem Phys. Lett. 2004, 393, 51-57.

[74] Volkov, A.; Coppens, P. Critical examination of the radial functions in the Hansen-Coppens multipole model through topological analysis of primary and refined theoretical densities. Acta Cysrat. 2001, A57, 395-405.

[75] Koritsanszky, T. Charge Density Refinements using the Standard and Upgraded Pseudoatom Model. 5th European Charge Density Meeting, Gravedona, 2008.

[76] Santos, L. H. R.; Macchi, P. to be submitted

[77] Pisani, C.; Maschio, L.; Casassa, S.; Halo, M. Schütz, M.; Usvyat, D. Periodic local MP2 method for the study of electronic correlation in crystals: Theory and preliminary applications. J. Comput. Chem., 2008 29, 2113-2124.

[78] Dovesi, R.; Saunders, V.R.; Roetti, C.; Orlando, R.; Zicovich-Wilson, C.M.; Pascale, F.; Civalleri, B.; Doll, K.; Harrison, N.M.; Bush, I.J.; D’Arco, P.; Llunell M. CRYSTAL09 User's Manual, Università di Torino, Torino, 2009.

[79] Giannozzi, P.; Baroni, S.; Bonini, N.; Calandra, M.; Car, R.; Cavazzoni, C.; Ceresoli, D.; Chiarotti, G.L.; Cococcioni, M.; Dabo, I.; Dal Corso, A.; Fabris, S.; Fratesi, G.; de Gironcoli, S.; Gebauer, R.; Gerstmann, U.; Gougoussis, C.; Kokalj, A.; Lazzeri, M.; Martin-Samos, L.; Marzari, N.; Mauri, F.; Mazzarello, R.; Paolini, S.; Pasquarello, A.; Paulatto, L.; Sbraccia, S.; Scandolo, C.; Sclauzero, G.; Seitsonen, A.P.; Smogunov, A.; Umari, P.; Wentzcovitch R.M. QUANTUM ESPRESSO: a modular and open-source software project for quantum simulations of materials. J. Phys.: Condens. Matter 2009, 21, 395502.

[80] Kresse, G.; Furthmüller, J. Efficiency of ab initio total-energy calculations for metals and semiconductors using a plane-wave basis set. Comput. Mater. Sci. 1996, 6, 15-50.

[81] a) Blaha, P.; Schwarz, K.; Madsen, G.; Kvasnicka, D.; Luitz, J. WIEN2k (current version WIEN2k_10.1), Inst. für Materials Chemistry, TU Vienna, 2010.

[82] Bader, R.F.W. Atoms in Molecules: A Quantum Theory. International Series of Monographs on Chemistry 22, Oxford Science Publications, Oxford 1990.

[83] Schwinger, J. Field theory of particles. In Johnson et all (eds), Brandeis Summer Institute in Theoretical Physics, Lectures on Particles and Field Theory. Englewood Cliffs, NJ, 1964.

[84] Bader, R.F.W.; Essén, H. The characterization of atomic interactions. J. Chem. Phys. 1984, 80, 1943-1960.

[85] See for example, Cassam-Chenäi, P.; Jayatilaka, D. Some fundamental problems with zero flux partitioning of electron densities. Theor. Chem. Acc. 2001, 105, 213-218.

[86] Popelier, P. L. A. Atoms in Molecules: An Introduction, Prentice Hall, 2000.

[87] Bader, R. F. W.; Beddal, P. M. Virial field relationship for molecular charge distributions and spatial partitioning of molecular properties. J. Chem. Phys. 1972, 56, 3320-3329.

[88] a) Gadre, S. R.; Shrivastava. I. H. Shapes and sizes of molecular anions via topographical analysis of electrostatic potential. J. Chem. Phys. 1991, 94, 4384-4391; b) Bouhmaida, N.; Dutheil, M.; Ghermani, N. E.; Becker, P. Gradient vector field and properties of the experimental electrostatic potential: Application to ibuprofen drug molecule. J. Chem. Phys. 2002, 116, 6196-6204; c) Mata, I.; Molins, E.; Alkorta, I.; Espinosa, E. Topological Properties of the Electrostatic Potential in Weak and Moderate N $\cdots$ H Hydrogen Bonds. J. Phys. Chem. 2007, 111, 6425-6433. 
[89] Pendàs, A. M.; Hernandez-Trujillo, J. The Ehrenfest force field: Topology and consequences for the definition of an atom in a molecule. J. Chem. Phys. 2012, 137, 134101-8.

[90] Iversen, B. B.; Larsen, F. K.; Souhassou, M.; Masaki, T. Experimental evidence for the existence of nonnuclear maxima in the electron-density distribution of metallic beryllium. A comparative study of the maximum entropy method and the multipole refinement method. Acta Cryst. 1995, B51, 580-591.

[91] Cao, W. L.; Gatti, C.; MacDougall, P. J.; Bader, R. F. W. On the presence of non-nuclear attractors in the charge distributions of Li and Na clusters. Chem. Phys. Lett. 1987, 141, 380-385.

[92] Silvi, B.; Gatti, C. Direct Space Representation of the Metallic Bond. J. Chem. Phys. 2000, 104, 947-953.

[93] Martín Pendás, A.; Blanco, M. A.; Costales, A.; Mori Sánchez, P.; Luaña, V. Non-nuclear Maxima of the Electron Density. Phys. Rev. B 1999, 83, 1930-1933

[94] Collard, K.; Hall, G.G. Orthogonal trajectories of the electron density Int. J. Quantum Chem., 1977, 12, 623-637

[95] Bader, R. F. W. A Bond Path: A Universal Indicator of Bonded Interactions. J. Phys. Chem. 1998, 102, 7314-7323

[96] a) Cioslowski, J.; Edgington, L.; Stefanov, B. B Steric Overcrowding in Perhalogenated Cyclohexanes, Dodecahedranes, and [60]Fulleranes. J. Am. Chem. Soc. 1995, 117, 10381-10384; b) Abramov, Y. A. Secondary Interactions and Bond Critical Points in Ionic Crystals. J. Phys. Chem. A 1997, 101, 5725-5728.

[97] a) Macchi, P.; Sironi, A. Chemical bonding in transition metal carbonyl clusters: complementary analysis of theoretical and experimental electron densities. Coord. Chem. Rev. 2003, 238-239, 383-412; b) Gatti, C. Chemical bonding in crystals: new directions. Z. Krist. 2005, 220, 399-457; c) Macchi, P.; Sironi, A. Interactions Involving Metals: From "Chemical Categories" to QTAIM, and Backwards, Chapter 13 in The Quantum Theory of Atoms in Molecules: From Solid State to DNA and Drug Design Matta, C. F. and Boyd, R. J. Eds, Wiley-VCH, 2007; d) Macchi, P. Electron Density Distribution in Organometallic Materials. Chimia, 2009, 63, 1-6; e) Espinosa, E.; Souhassou, M.; Lachekar, H.; Lecomte, C. Topological analysis of the electron density in hydrogen bond. Acta Cryst 1999, B55, 563-572; f) Espinosa, E.; Alkorta, I.; Elguero, J.; Molins, E. From weak to strong interactions: A comprehensive analysis of the topological and energetic properties of the electron density distribution involving X-H center dot center dot center dot F-Y systems. J. Chem. Phys. 2002, 117, 5529-5542; g) Pendas, A.M.; Francisco, E.; Blanco, M.A.; Gatti, C. Bond paths as Privileged Exchange Channels. Chem. Eur. J. 2007, 12, 9362-9371.

[98] Bader, R.F.W.; Stephens, M.E. Fluctuation and correlation of electrons in molecular sys-tems. Chem. Phys. Lett. 1974, $26,445-449$.

[99] Blanco, M.A.; Martín Pendás, A.; Francisco, E. Interacting Quantum Atoms: A Correlated Energy Decomposition Scheme Based on the Quantum Theory of Atoms in Molecules. J. Chem. Theory Comput. 2005, 1, 1096-1109.

[100] Becke, A. D.; Edgecombe, K. E. A simple measure of electron localization in atomic and molecular systems. J. Chem. Phys. 1990, 92 , 5397-5403.

[101] Kohout, M. A Measure of Electron Localizability. Int. J. of Quant. Chem. 2004, 97, 651-658.

[102] Martín Pendás, A.; Kohout, M.; Blanco, M. A.; Francisco, E. Beyond Standard Charge Density Topological

Analyses in Gatti, C.; Macchi, P. editors Modern charge density analysis. Springer. 2012.

[103] Bader, R.F.W.; Gatti, C. A Green's function for the density. Chem. Phys. Lett. 1998, 287, 233-238.

[104] Arfken, G. Mathematical Methods for Physicists. Academic Press, Orlando, Florida, 1985.

[105] Gatti, C. The Source Function Descriptor as a Tool to Extract Chemical Information from Theoretical and Experimental Electron Densities, Struct. Bond. 2012, 147, 193-286.

[106] Farrugia, L.J.; Macchi, P. On the Interpretation of the Source Function. J. Phys. Chem. A 2009, 113, 10058-10067.

[107] Spackman, M. A.; Maslen, E. N. Electron Density and the Chemical Bond. A reappraisal of Berlin's Theorem. Acta Cryst. 1985, A41, 347-353.

[108] Berlin, T. Binding Regions in Diatomic Molecules. J. Chem. Phys. 1951, 19, 208-213.

[109] a) Parr, R.G.; Yang, W. Density-Functional Theory of Atoms and Molecules. Oxford UP, New York, 1989; b) Geerlings, P.; De Proft, F.; Langenaeker, W. Conceptual density functional theory. Chem. Rev. 2003, 103, 1793-1873.

[110] Spackman, M.A.; Byrom, P.G. A novel definition of a molecule in a crystal. Chem. Phys. Lett. 1997, 267, 215-220.

[111] Spackman, M.A.; Jayatilaka, D. Hirshfeld surface analysis. CrystEngComm 2009, 11, 19-32.

[112] a) Stewart, R. F. Mapping electrostatic potentials from diffraction data. God. Jugosl. Cent. Kristalogr. 1982, 17, 1-24; b) Stewart, R.F.; Craven, B. M. Molecular Electrostatic Potentials From Crystal Diffraction - The Neurotransmitter Gamma-Aminobutyric-Acid. Biophys. J.,1993, 65, 998-1005; c) Stewart, R. F. Electrostatic properties of molecules from diffraction data. In: Jeffrey, G.A., Piniella, J.F. (eds.) The Application of Charge Density Research to Chemistry and Drug Design, pp 63-101, NATO ASI Ser. B 250, 1991. Plenum Publishing Corp., New York; d) Volkov, A.; King, H.F.; Coppens, P.; Farrugia, L.J. On the calculation of the electrostatic potential, electric field and electric field gradient from the aspherical pseudoatom model. Acta Cryst. 2006, A62, 400-408.

[113] Buckingham, A.D. Permanent and Induced Molecular Moments and Long-Range Intermolecular Forces. Adv. Chem. Phys. 1967, 12, 107-142.

[114] Price, S. L. Theoretical approaches to the study of non-bonded interactions. NATO Science Series E: Applied Sciences. 1999, 360 223-234.

[115] a) Spackman, M. A. Atom-Atom potential via electron gas theory. J. Chem. Phys, 1986, 185, 6579-6586; b) Spackman, M. A. A simple quantitative model of hydrogen bonding. J. Chem. Phys., 1986, 85, 6587-6601; c) Spackman, M. A. A simple quantitative model of hydrogen bonding. Application to more complex systems. J. Phys. Chem., 1987, 91, 3179-3186.

[116] Spackman, M. A. The use of promolecular density to approximate the penetration contribution to intermolecular energies. Chem. Phys. Lett., 2005, 418, 158-162. 
[117] Volkov, A.; Koritsanszky, T.; Coppens, P. Combination of the exact potential and multipole methods (EP/MM) for evaluation of intermolecular electrostatic interaction energies with pseudoatom representation of molecular electron densities. Chem. Phys.Lett. 2004, 391, 170-175.

[118] Gavezzotti, A. Calculation of Intermolecular Interaction Energies by Direct Numerical Integration over Electron Densities. I. Electrostatic and Polarization Energies in Molecular Crystals. J. Phys. Chem., 2002 106, 4145-4154 (2002)

[119] Volkov, A.; Li, X.; Koritsanszky, T.; Coppens, P. Ab Initio Quality Electrostatic Atomic and Molecular Properties Including Intermolecular Energies from a Transferable Theoretical Pseudoatom Databank. J. Phys. Chem. A 2004, 108, 4283-4300.

[120] Lennard-Jones, J. E. On the Determination of Molecular Fields. Proc. R. Soc. Lond. A 1924, 106, 463-477.

[121] Williams, G. J.; Stone, A. J. Distributed dispersion: A new approach. J. Chem. Phys. 2003, 119, 4620-4628.

[122] Abramov, Y. A.; Volkov, A.; Wu, G.; Coppens, P. Use of X-ray charge densities in the calculation of intermolecular interactions and lattice energies: Application to glycylglycine, dl-histidine, and dl-proline and comparison with theory. J. Phys. Chem. B 2000, 104, 2183-2188.

[123]London, F. On the Theory and Systematic of Molecular Forces. Z. Phys. 1930, 63, 245-279.

[124] Lippincott, E. R.; Schroeder, R. One-Dimensional Model Of The Hydrogen Bond. J. Chem. Phys. 1955, 23, 10991106.

[125] Espinosa, E.; Molins, E.; Lecomte, C. Hydrogen bond strengths revealed by topological analyses of experimentally observed electron densities. Chem. Phys.Lett.1998, 285, 170-173.

[126] Macchi, P.; Iversen, B.B; Sironi, A.; Chakoumakos, B.C; Larsen, F.K. Interanionic O - H $\cdots$ O interactions: The charge density point of view. Angew. Chem. 2000, 39, 2719-2722.

[127] Chimpri, A. S.; Evans, S. R.; Casati, N. Macchi, P. manuscript in preparation.

[128] Cummins, P. G.; Dunmur, D. A.; Munn, R. W.; Newham, R: J. Applications Of Ewald Method .2. Homogeneous Strain Derivatives Of Dipole Lattice Sums. Acta Cryst. 1976, A32, 854-858.

[129] Dunmur, D. A. Local Electric-Field In Anisotropic Molecular-Crystals. Mol. Phys. 1972, 23, 109-115.

[130] Ewald, P. P. Die Berechnung optischer und elektrostatischer Gitterpotentiale. Ann. Phys. 1921, 369, 253-287.

[131] Whitten, A. E.; Jayatilaka, D.; Spackman, M. A. Effective molecular polarizabilities and crystal refractive indices estimated from x-ray diffraction data. J. Chem. Phys. 2006, 125, 174505.

[132] Poater, J.; Solà, M.; Miquel, D.; Fradera, X. The calculation of electron localization and delocalization indices at the Hartree-Fock, density functional and post-Hartree-Fock levels of theory. Theor. Chem. Acc. 2002, 107, 362-371 